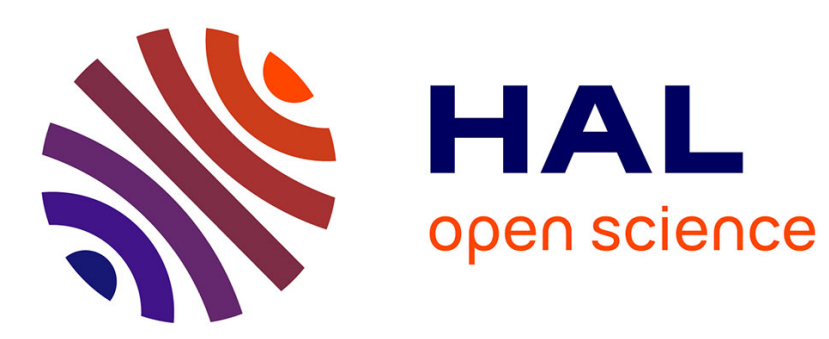

\title{
Mirrored Variants of the (1,2)-CMA-ES Compared on the Noisy BBOB-2010 Testbed
}

\author{
Anne Auger, Dimo Brockhoff, Nikolaus Hansen
}

\section{To cite this version:}

Anne Auger, Dimo Brockhoff, Nikolaus Hansen. Mirrored Variants of the (1,2)-CMA-ES Compared on the Noisy BBOB-2010 Testbed. GECCO workshop on Black-Box Optimization Benchmarking (BBOB'2010), Jul 2010, Portland, OR, United States. pp.1575-1582, 10.1145/1830761.1830775 . inria-00502436

\section{HAL Id: inria-00502436 \\ https://hal.inria.fr/inria-00502436}

Submitted on 14 Jul 2010

HAL is a multi-disciplinary open access archive for the deposit and dissemination of scientific research documents, whether they are published or not. The documents may come from teaching and research institutions in France or abroad, or from public or private research centers.
L'archive ouverte pluridisciplinaire HAL, est destinée au dépôt et à la diffusion de documents scientifiques de niveau recherche, publiés ou non, émanant des établissements d'enseignement et de recherche français ou étrangers, des laboratoires publics ou privés. 


\title{
Mirrored Variants of the (1,2)-CMA-ES Compared on the Noisy BBOB-2010 Testbed
}

\author{
[Black-Box Optimization Benchmarking Workshop] \\ Anne Auger, Dimo Brockhoff, and Nikolaus Hansen \\ Projet TAO, INRIA Saclay-lle-de-France \\ LRI, Bât 490, Univ. Paris-Sud \\ 91405 Orsay Cedex, France \\ firstname.lastname@inria.fr
}

\begin{abstract}
Derandomization by means of mirrored samples has been recently introduced to enhance the performances of $(1, \lambda)$ and $(1+2)$ Evolution-Strategies (ESs) with the aim of designing fast local search stochastic algorithms. In this paper, we investigate the impact of mirrored samples for noisy optimization. Since elitist selection is detrimental for noisy optimization, we investigate non-elitist ESs only here. We compare on the BBOB-2010 noisy benchmark testbed two variants of the $(1,2)$-CMA-ES where mirrored samples are implemented with the baseline (1,2)-CMA-ES. Each algorithm implements a restart mechanism. A total budget of $10^{4} \mathrm{D}$ function evaluations per trial has been used, where $D$ is the dimension of the search space.

The experiments clearly show a ranking among the three algorithms: both mirroring variants have lower expected running times than the (1,2)-CMA-ES by at least $50 \%$ on 5 functions and they solve three additional functions in 20D that the (1,2)-CMA-ES cannot solve (or only with small probability). The comparison between the two mirroring variants is in favor of the algorithm employing a sequential selection in addition - outperforming the algorithm with only mirrored samples on five functions by at least $17 \%$ whereas no statistically significant worsening can be observed. Both algorithms using mirrored samples also outperform the function-wise best algorithm of the BBOB-2009 benchmarking on three (respectively four) functions comprising Cauchy noise by up to $65 \%$.
\end{abstract}

\section{Categories and Subject Descriptors}

G.1.6 [Numerical Analysis]: Optimization-global optimization, unconstrained optimization; F.2.1 [Analysis of Algorithms and Problem Complexity]: Numerical Algorithms and Problems

(C)ACM, 2010. This is the authors' version of the work. It is posted here by permission of ACM for your personal use. Not for redistribution. The definitive version was published at GECCO'10, July 7-11, 2010, Portland, OR, USA. http://doi.acm.org/10.1145/1830761.1830775
General Terms

Algorithms

\section{Keywords}

Benchmarking, Black-box optimization

\section{INTRODUCTION}

Evolution Strategies (ESs) are robust stochastic search algorithms for black-box optimization where the function to be minimized, $f$, maps the continuous search space $\mathbb{R}^{D}$ into R. ESs evolve a population of candidate solutions that are created by sampling $\lambda$ independent random vectors following a multivariate normal distribution. Recently, a new derandomization technique replacing the independent sampling of new solutions (or offspring) by mirrored samples has been introduced to enhance the performances of ESs [1]. With mirrored sampling, a single sample $\mathcal{N}$ of a multivariate normal distribution is used for two offspring of the same iteration. Denoting $X$ the current solution, the two offspring will equal $X+\mathcal{N}$ and $X-\mathcal{N}$ respectively. The resulting offspring are thus symmetric or mirrored with respect to $X$ and are thus negatively correlated. Mirrored samples have been implemented in the Covariance-Matrix-Adaptation EvolutionStrategy (CMA-ES), an ES whose characteristic is to adapt the full covariance matrix of the multivariate normal search distribution [6]. Another new concept called sequential selection was introduced together with mirrored samples [1]. Sequential selection, consists in performing sequential evaluations of the offspring and breaking the evaluation loop as soon as an offspring is better than the current solution $X$ and thus saving the remaining fitness evaluations.

In this paper, we assess quantitatively the improvement that can be brought by mirrored samples and by mirrored samples coupled with sequential selection. We compare on the BBOB-2010 noisy testbed the $(1,2)$-CMA-ES with two variants: first the $\left(1,2_{\mathrm{m}}\right)$-CMA-ES where mirrored samples are used, and second the $\left(1,2_{\mathrm{m}}^{\mathrm{s}}\right)$-CMA-ES that in addition to the mirrored samples uses sequential selection. The algorithms and the CPU timing experiments are described in a complementing paper in the same proceeding [2]. 


\section{RESULTS}

\subsection{Comparing (1,2)- and $\left(1,2_{\mathrm{m}}\right)$-CMA-ES}

Results from experiments comparing $(1,2)$-CMA-ES and (1,2 $\mathrm{m}$-CMA-ES according to [4] on the benchmark functions given in $[3,5]$ are presented in Figures 1 and 2 and in Table 1. The expected running time (ERT), used in the figures and table, depends on a given target function value, $f_{\mathrm{t}}=$ $f_{\text {opt }}+\Delta f_{\mathrm{t}}$, and is computed over all relevant trials as the number of function evaluations executed during each trial while the best function value did not reach $f_{\mathrm{t}}$, summed over all trials and divided by the number of trials that actually reached $f_{\mathrm{t}}[4,7]$. Statistical significance is tested with the rank-sum test for a given target $\Delta f_{\mathrm{t}}$ using, for each trial, either the number of needed function evaluations to reach $\Delta f_{\mathrm{t}}$ (inverted and multiplied by -1 ), or, if the target was not reached, the best $\Delta f$-value achieved, measured only up to the smallest number of overall function evaluations for any unsuccessful trial under consideration.

According to the experiments, the $(1,2 \mathrm{~m})$-CMA-ES clearly outperforms the baseline algorithm $(1,2)$-CMA-ES. Both in $5 \mathrm{D}$ and in $20 \mathrm{D}$, the $\left(1,2_{\mathrm{m}}\right)$-CMA-ES is on no function and for no target $\leq 1$ worse than the $(1,2)$-CMA-ES. Moreover, statistically significant improvements can be reported on 8 and 11 functions in 20D and 5D respectively. The improvement factors are ranging from about 1.8 for $f_{101}$ and $f_{103}$ over 2-3 $\left(f_{106}\right.$ and $\left.f_{109}\right)$ to a factor of more than 5 for the sphere function with moderate uniform noise $\left(f_{102}\right.$, all in $\left.20 \mathrm{D}\right)$. In $20 \mathrm{D}$, three functions can be solved for all 15 instances by the $\left(1,2_{\mathrm{m}}\right)$-CMA-ES whereas the (1,2)-CMA-ES has a lower (on $f_{106}$ ) or much lower success probability (only 1 out of 15 instances were solved by the $(1,2)$-CMA-ES on $f_{112}$ and 0 instances were solved for $\left.f_{118}\right)$. Similar results hold for 5D.

On $f_{109}$ (in both 5D and 20D and for several difficult targets), on $f_{130}$ (in $20 \mathrm{D}$ and for a target of $10^{-7}$ ), as well as on medium targets on $f_{121}$, the $\left(1,2_{\mathrm{m}}\right)$-CMA-ES outperforms the function-wise best algorithm of the BBOB-2009 benchmarking. The largest improvement is achieved for the Gallagher function with Cauchy noise $\left(f_{130}\right)$ where the expected running time of the $\left(1,2_{\mathrm{m}}\right)$-CMA-ES is about $40 \%$ lower than for the best algorithm of BBOB-2009 for that function of last year's benchmarking.

\section{$2.2\left(1,2_{m}\right)-$ and $\left(1,2_{m}^{\mathrm{s}}\right)-\mathrm{CMA}-\mathrm{ES}$}

The results of this comparison are shown in Fig. 3 and 4 and in Table 2.

In $5 \mathrm{D}$, no statistically significant differences (except for $f_{121}$ where the $\left(1,2_{\mathrm{m}}^{\mathrm{s}}\right)$-CMA-ES is outperforming the $\left(1,2_{\mathrm{m}}\right)$ CMA-ES) can be observed. In 20D, however, the $\left(1,2_{\mathrm{m}}^{\mathrm{s}}\right)$ CMA-ES clearly shows a better (statistically significant) performance than the $(1,2 \mathrm{~m})$-CMA-ES on 6 functions: on $f_{101}$, the improvement is $17 \%$, on $f_{102}$ and $f_{103}$ about $20 \%$, on $f_{109} 25 \%$, on $f_{112}$ about $60 \%$, and on $f_{118}$ about $30 \%$.

The $\left(1,2_{\mathrm{m}}^{\mathrm{s}}\right)$-CMA-ES outperforms the function-wise best algorithm of BBOB-2009 on $f_{103}$ by about $12 \%$, on $f_{109}$ and $f_{112}$ by about $30 \%$, and on $f_{130}$ by about $65 \%$ for several low target values in 20D.

\subsection{Comparing (1,2)- and $\left(1,2_{\mathrm{m}}^{\mathrm{s}}\right)$-CMA-ES}

The third comparison yields similar results for the $\left(1,2_{\mathrm{m}}^{\mathrm{s}}\right)$ CMA-ES than for the $(1,2 \mathrm{~m})$-CMA-ES above when compared to the $(1,2)$-CMA-ES, except that the improvement over the (1,2)-CMA-ES is even larger - not surprisingly af- ter the results of Sec. 2.2. In 20D (5D), the (1,2)-CMA-ES is outperformed by the $\left(1,2_{\mathrm{m}}^{\mathrm{s}}\right)$-CMA-ES on 8 (12) functions (with statistical significance). The expected running time of the $\left(1,2_{\mathrm{m}}^{\mathrm{s}}\right)$-CMA-ES is always smaller than the one of the (1,2)-CMA-ES (with a few exceptions in 2D and 3D, cp. 3). Note that due to space limitations, we cannot show the plots and tables of this comparison.

\section{CONCLUSIONS}

The idea behind derandomization by means of mirroring introduced in [1] is to use only one random sample from a multivariate normal distribution to create two (negatively correlated or mirrored) offspring. Thereby, the first offspring is generated by adding a random sample to the parent solution and the second offspring then equals the solution which is symmetric to the first offspring with respect to the parent (by adding the negative sample to the parent). Here, this concept of mirroring has been integrated within two variants of a simple (1,2)-CMA-ES (of which the $\left(1,2_{\mathrm{m}}^{\mathrm{s}}\right.$ )CMA-ES uses sequential selection [1] in addition and the (1,2 $)$-CMA-ES does not). The three algorithms are then compared on the noisy BBOB-2010 testbed.

The experiments clearly show a ranking among the three algorithms: both the $(1,2 \mathrm{~m})$-CMA-ES and the $\left(1,2_{\mathrm{m}}^{\mathrm{s}}\right)$-CMAES are never worse than the (1,2)-CMA-ES and have lower expected running times than the baseline by at least $50 \%$ on 5 functions. Moreover, the two algorithms employing mirroring solve three additional functions in 20D which the $(1,2)$-CMA-ES cannot solve (or only with small probability). The comparison between the $\left(1,2_{\mathrm{m}}\right)$-CMA-ES and the $\left(1,2_{\mathrm{m}}^{\mathrm{s}}\right)$-CMA-ES is in favor of the $\left(1,2_{\mathrm{m}}^{\mathrm{s}}\right)$-CMA-ES outperforming the $\left(1,2_{\mathrm{m}}\right)$-CMA-ES on five functions by at least $17 \%$ whereas no statistically significant worsening can be observed. Both the $\left(1,2_{\mathrm{m}}\right)$-CMA-ES and the $\left(1,2_{\mathrm{m}}^{\mathrm{s}}\right)$-CMAES also outperform the function-wise best algorithm of the BBOB-2009 benchmarking on three (respectively four) functions comprising Cauchy noise by up to $65 \%$.

\section{Acknowledgments}

This work receives support by the French national research agency (ANR) within the SYSCOMM project ANR-08-SYSC017 and within the COSINUS project ANR-08-COSI-007-12.

\section{REFERENCES}

[1] A. Auger, D. Brockhoff, and N. Hansen. Mirrored sampling and sequential selection for evolution strategies. Rapport de Recherche RR-7249, INRIA Saclay- ̂lle-de-France, April 2010.

[2] A. Auger, D. Brockhoff, and N. Hansen. Mirrored variants of the (1,2)-CMA-ES compared on the noiseless BBOB-2010 testbed. In GECCO (Companion), 2010.

[3] S. Finck, N. Hansen, R. Ros, and A. Auger. Real-parameter black-box optimization benchmarking 2010: Presentation of the noisy functions. Technical Report 2009/21, Research Center PPE, 2010.

[4] N. Hansen, A. Auger, S. Finck, and R. Ros. Real-parameter black-box optimization benchmarking 2010: Experimental setup. Technical Report RR-7215, INRIA, 2010.

[5] N. Hansen, S. Finck, R. Ros, and A. Auger. Real-parameter black-box optimization benchmarking 
101 Sphere (moderate) 104 Rosenbrock (moderate)

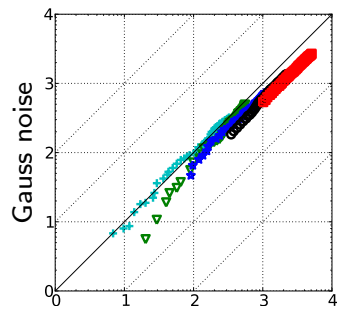

102 Sphere (moderate)

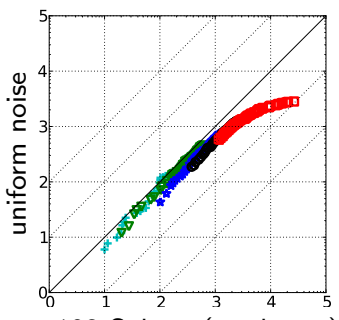

103 Sphere (moderate)

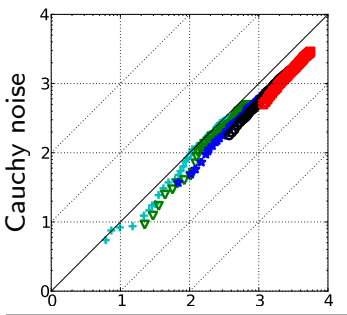

116 Ellipsoid

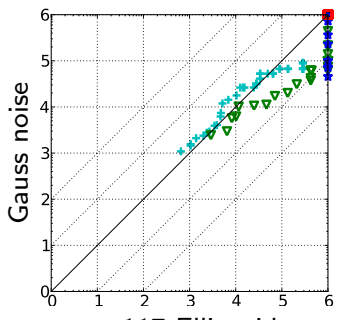

117 Ellipsoid

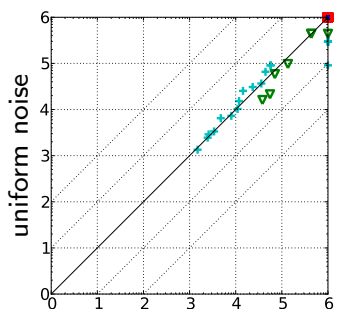

118 Ellipsoid

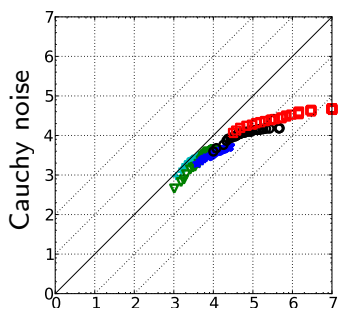

107 Sphere

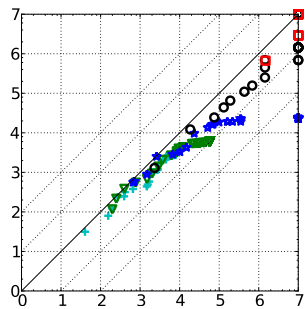

105 Rosenbrock (moderate)

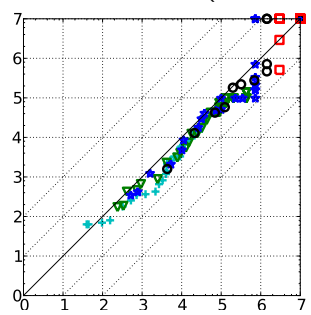

106 Rosenbrock (moderate)

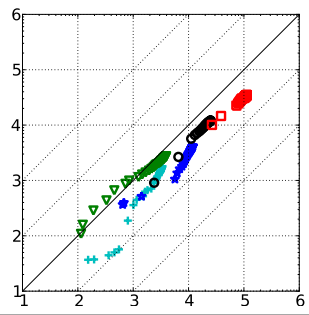

119 Sum of diff powers

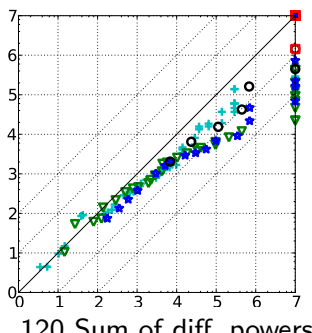

120 Sum of diff. powers

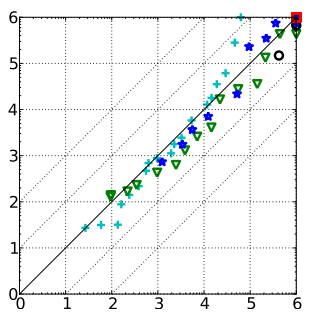

121 Sum of diff. powers

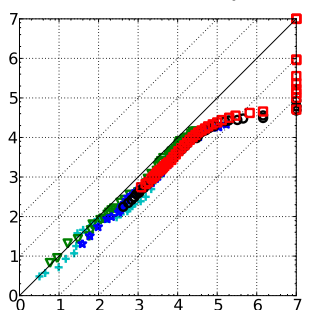

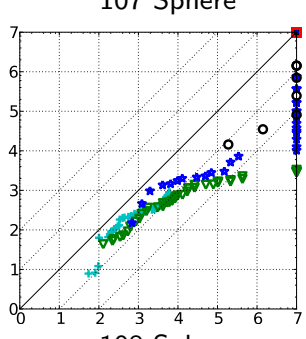

108 Sphere

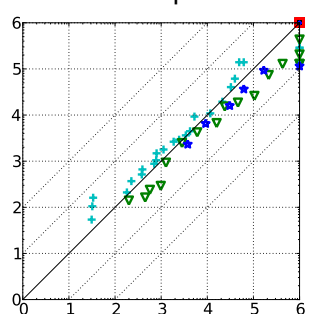

109 Sphere

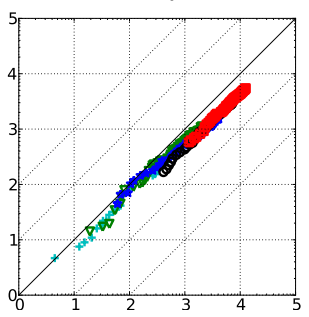

122 Schaffer F7

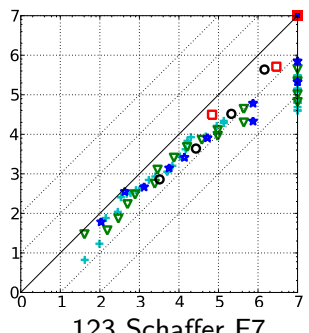

123 Schaffer F7

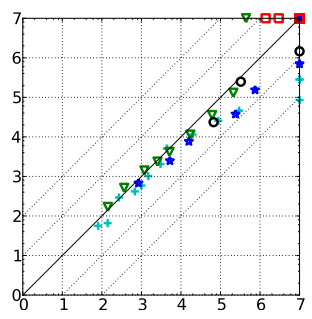

124 Schaffer F7

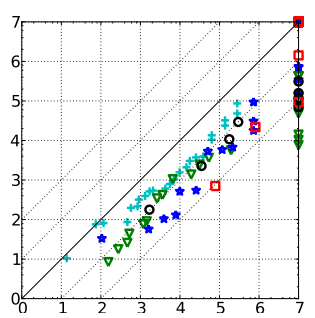

110 Rosenbrock

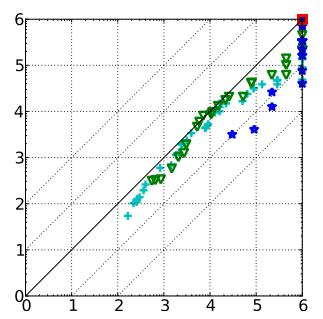

111 Rosenbrock

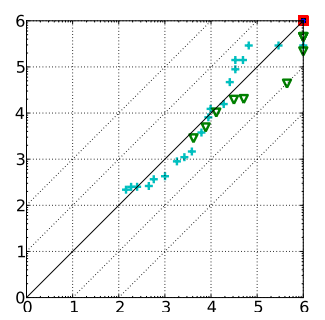

112 Rosenbrock

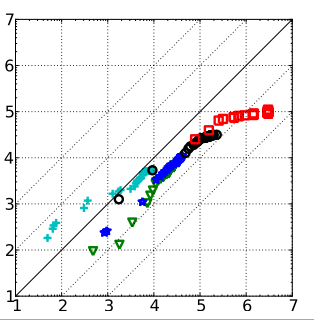

125 Griewank-Rosenbrock

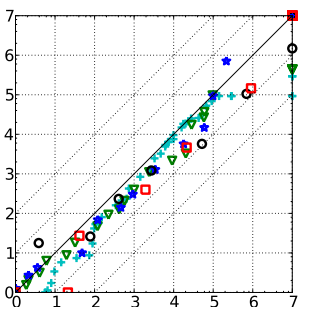

126 Griewank-Rosenbrock

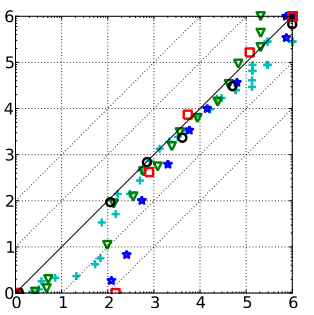

127 Griewank-Rosenbrock

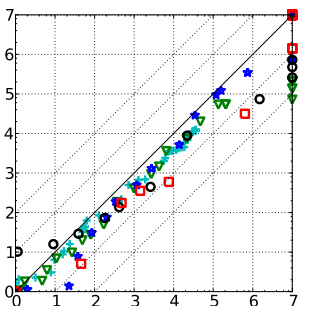

113 Step-ellipsoid

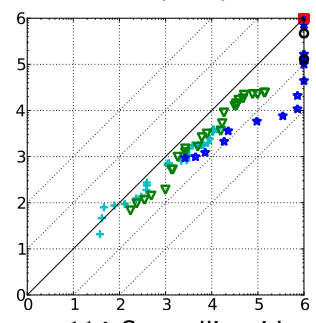

114 Step-ellipsoid

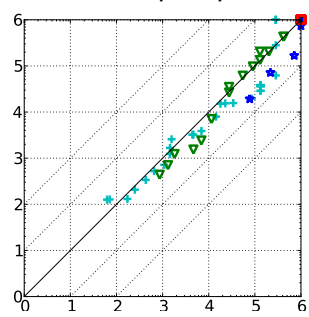

115 Step-ellipsoid

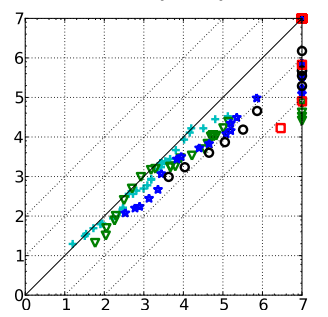

128 Gallagher

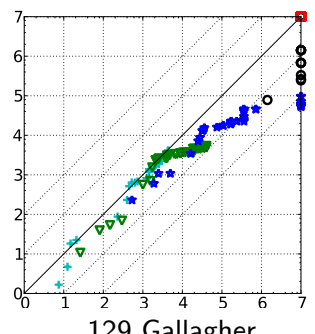

129 Gallagher

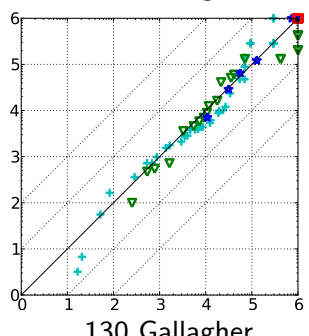

130 Gallagher

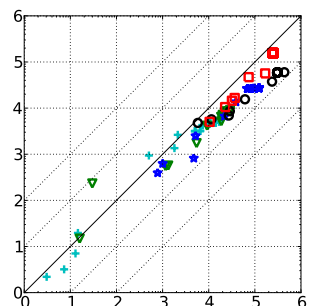

Figure 1: Expected running time (ERT in log10 of number of function evaluations) of (1,2 $\mathrm{m}$ )-CMA-ES versus $(1,2)-C M A-E S$ for 46 target values $\Delta f \in\left[10^{-8}, 10\right]$ in each dimension for functions $f_{101}-f_{130}$. Markers on the upper or right edge indicate that the target value was never reached by $\left(1,2_{\mathrm{m}}\right)$-CMA-ES or (1,2)-CMA-ES respectively. Markers represent dimension: 2: $+, 3: \nabla, 5: \star, 10: \circ, 20: \square$. 
$5-\mathrm{D}$
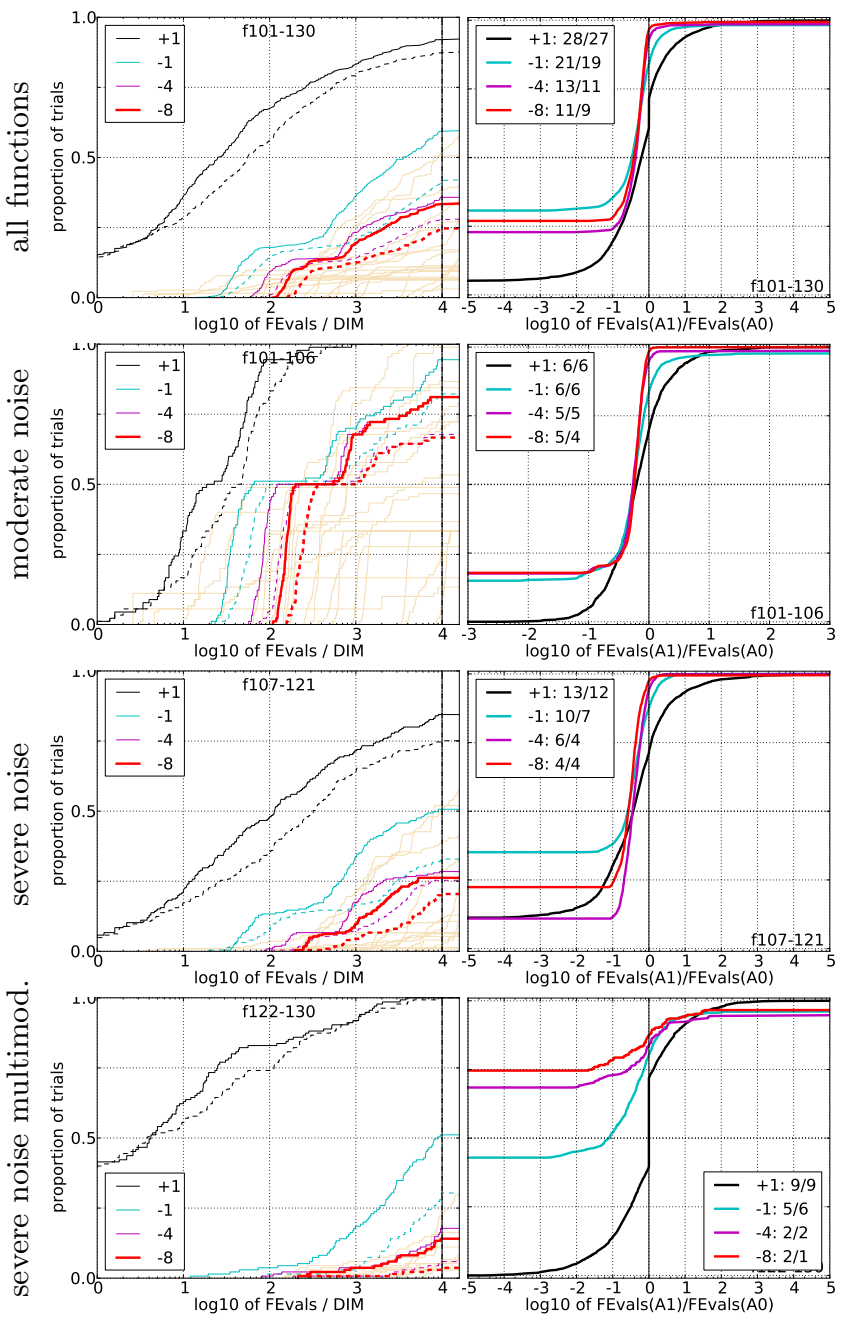

$20-\mathrm{D}$
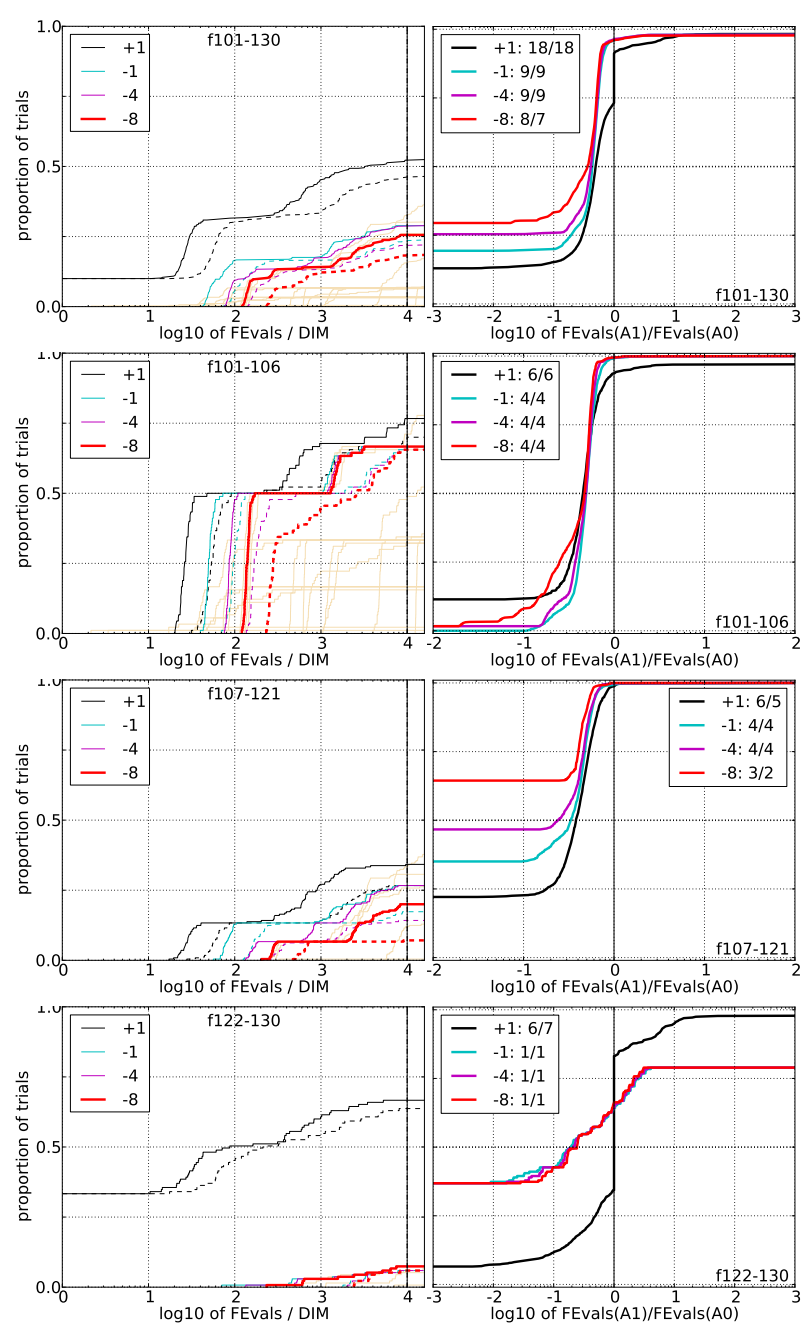

Figure 2: Empirical cumulative distributions (ECDF) of run lengths and speed-up ratios in 5-D (left) and 20-D (right). Left sub-columns: ECDF of the number of necessary function evaluations divided by dimension $D$ (FEvals/D) to reached a target value $f_{\text {opt }}+\Delta f$ with $\Delta f=10^{k}$, where $k \in\{1,-1,-4,-8\}$ is given by the first value in the legend, for $\left(1,2_{\mathrm{m}}\right)$-CMA-ES (solid) and (1,2)-CMA-ES (dashed). Light beige lines show the ECDF of FEvals for target value $\Delta f=10^{-8}$ of all algorithms benchmarked during BBOB-2009. Right sub-columns: ECDF of FEval ratios of $\left(1,2_{\mathrm{m}}\right)$-CMA-ES divided by $(1,2)$-CMA-ES, all trial pairs for each function. Pairs where both trials failed are disregarded, pairs where one trial failed are visible in the limits being $>0$ or $<1$. The legends indicate the number of functions that were solved in at least one trial $\left(\left(1,2_{\mathrm{m}}\right)-\mathrm{CMA}-\mathrm{ES}\right.$ first $)$. 
$5-\mathrm{D}$

\begin{tabular}{|c|c|c|c|c|c|c|c|}
\hline$\Delta f$ & $1 \mathrm{e}+1$ & $1 \mathrm{e}+0$ & $1 e-1$ & $1 e-3$ & $1 e-5$ & $1 e-7$ & \#succ \\
\hline$f_{101}$ & 11 & 37 & 44 & 62 & 69 & 75 & $15 / 15$ \\
\hline$(1,2)-C M A-E S$ & 8.3 & 4.4 & 6.3 & 8.5 & 11 & 13 & $15 / 15$ \\
\hline$(1,2 \mathrm{~m})$-CMA-ES & 4.3 & 3.3 & 4.9 & 5.8 & $7.4^{\star}$ & $8.9^{\star 2}$ & $15 / 15$ \\
\hline$f_{102}$ & 11 & 35 & 50 & 72 & 86 & 99 & $15 / 15$ \\
\hline$(1,2)$-CMA-ES & 9.2 & 5.8 & 6.2 & 7.7 & 9.7 & 11 & $15 / 15$ \\
\hline$(1,2 \mathrm{~m})$-CMA-ES & 3.9 & 3.3 & $3.6^{\star}$ & $4.9^{\star 2}$ & $6^{\star 3}$ & $6.8^{\star 3}$ & $15 / 15$ \\
\hline$f_{103}$ & 11 & 28 & 30 & 31 & 35 & 120 & $15 / 15$ \\
\hline$(1,2)-C M A-E S$ & 6.2 & 6.7 & 9 & 18 & 25 & 10 & $15 / 15$ \\
\hline$(1,2 \mathrm{~m})$-CMA-ES & 3.4 & $3.8^{\star}$ & $5.5^{\star 2}$ & $10^{\star 3}$ & $15^{\star 3}$ & $6.1^{\star 3}$ & $15 / 15$ \\
\hline & 170 & 770 & 1300 & 1800 & 2000 & 2300 & $15 / 15$ \\
\hline & 3.9 & 13 & 54 & $\infty$ & $\infty$ & $\infty 5.0 e 4$ & $0 / 15$ \\
\hline
\end{tabular}

\begin{tabular}{c|cccccc|c}
$(1,2 \mathrm{~m})-\mathrm{CMA}-\mathrm{ES}$ & 3.3 & 4.3 & 13 & $\mathbf{1 3}^{\star 3}$ & $\mathbf{1 2}^{\star 3}$ & $\mathbf{1 1}^{\star 3}$ & $13 / 15$ \\
\hline $\mathbf{f} \mathbf{1 0 5}$ & 170 & 1400 & 5200 & $1.0 \mathrm{e} 4$ & $1.1 \mathrm{e} 4$ & $1.1 \mathrm{e} 4$ & $15 / 15$ \\
$(1,2)-\mathrm{CMA}-\mathrm{ES}$ & 3.1 & 7.8 & 19 & 70 & $\infty$ & $\infty 5.0 e 4$ & $0 / 15$
\end{tabular}

\begin{tabular}{l|clcccc|c}
$(1,2 \mathrm{~m})$-CMA-ES & 3.1 & 7.8 & 19 & 70 & $\infty$ & $\infty 5.0 e 4$ & $0 / 15$ \\
\hline & 2.1 & 6 & 9.8 & 20 & $\infty$ & $\infty 5.0 e 4$ & $0 / 15$ \\
\hline
\end{tabular} \begin{tabular}{c|cccccc|c}
\hline $\mathbf{f}_{106}$ & 86 & 530 & 1100 & 2700 & 2900 & 3100 & $15 / 15$ \\
$(1,2)-\mathrm{CMA}-\mathrm{ES}$ & 7.5 & 13 & 8.6 & 4.1 & 3.9 & 3.8 & $15 / 15$ \\
\hline
\end{tabular} \begin{tabular}{c|llllll|l}
$(1,2 \mathrm{~m})$-CMA-ES & 4.3 & 3.1 & $\mathbf{2 . 5}^{\star 2}$ & $\mathbf{1 . 3}^{\star 3}$ & $\mathbf{1 . 3}^{\star 3}$ & $\mathbf{1 . 3}^{\star 3}$ & $15 / 15$ \\
\hline $\mathbf{f}_{\mathbf{1 0 7}}$ & 40 & 230 & 450 & 940 & 1400 & 1900 & $15 / 15$
\end{tabular} \begin{tabular}{l|ccccccc}
$(1,2)$-CMA-ES & 17 & 43 & 330 & $\infty$ & $\infty$ & $\infty 5.0 e 4$ & $0 / 15$ \\
$(1,2 \mathrm{~m})$-CMA-ES & 3.8 & $\mathbf{7 . 7}^{\star}$ & $\mathbf{6 . 7}$ & $\mathbf{7 6}^{\star 3}$ & $\infty$ & $\infty 5.0 e 4$ & $0 / 15$ \\
\hline
\end{tabular} \begin{tabular}{c|cccccc|c} 
f 108 & 87 & 5100 & $1.4 \mathrm{e} 4$ & $3.1 \mathrm{e} 4$ & $5.9 \mathrm{e} 4$ & $\infty 5.0 \mathrm{e} 4$ & $0 / 15$ \\
\hline$(1,2)-\mathrm{CMA}-\mathrm{e} 4$ & 43 & $\infty$ & $\infty$ & $\infty$ & $\infty$ & $\infty 5.0 \mathrm{e} 4$ & $0 / 15$ \\
\hline
\end{tabular} \begin{tabular}{c|cccccc|c}
$(1,2 \mathrm{~m})$-CMA-ES & 27 & \multicolumn{1}{c}{$\mathrm{f}_{109}$} & $\infty$ & $\infty$ & $\infty$ & $\infty 5.0 e 4$ & $0 / 15$ \\
\hline$(1,2)$-CMA-ES & 5.5 & 2.8 & 220 & 570 & 870 & 950 & $15 / 15$ \\
$(1,2$ & 1.9 & 2.5 & 3.4 & $15 / 15$
\end{tabular} \begin{tabular}{l|cccccc|c}
$(1,2)$-CMA-ES & 5.5 & 2.8 & 1.8 & 1.9 & 2.5 & 3.4 & $15 / 15$ \\
$(1,2 \mathrm{~m})$-CMA-ES & 4.1 & 2.5 & 1.2 & $\mathbf{1}^{\star}$ & $\mathbf{1}^{\star 2}$ & $\mathbf{1 . 3}^{\star 2}$ & $15 / 15$ \\
\hline
\end{tabular} \begin{tabular}{c|cccccc|c}
$\mathbf{f}_{110}$ & 950 & $3.4 \mathrm{e} 4$ & $1.2 \mathrm{e} 5$ & $5.9 \mathrm{e} 5$ & $6.0 \mathrm{e} 5$ & $6.1 \mathrm{e} 5$ & $15 / 15$ \\
$(1,2)-\mathrm{CMA}-\mathrm{ES}$ & 32 & $\infty$ & $\infty$ & $\infty$ & $\infty$ & $\infty 5.0 e 4$ & $0 / 15$ \\
\hline
\end{tabular} \begin{tabular}{c|cccccc|c}
$(1,2 \mathrm{~m})-\mathrm{CMA}-\mathrm{ES}$ & $\mathbf{3 . 4}^{\star}$ & $\mathbf{2 . 3}^{\star 2}$ & $\mathbf{2 . 8}^{\star 2}$ & $\infty$ & $\infty$ & $\infty 5.0 \mathrm{e} 4$ & $0 / 15$ \\
\hline $\mathbf{f}_{\mathbf{1 1 1}}$ & 6900 & $6.1 \mathrm{e} 5$ & $8.8 \mathrm{e} 6$ & $2.3 \mathrm{e} 7$ & $3.1 \mathrm{e} 7$ & $3.1 \mathrm{e} 7$ & $3 / 15$ \\
& $\infty$ & & & & &
\end{tabular} \begin{tabular}{l|cccccc|c}
$(1,2)$-CMA-ES & $\infty$ & $\infty$ & $\infty$ & $\infty$ & $\infty$ & $\infty 5.0 e 4$ & $0 / 15$ \\
$(1,2 \mathrm{~m})$-CMA-ES & $\infty$ & $\infty$ & $\infty$ & $\infty$ & $\infty$ & $\infty 5.0 e 4$ & $0 / 15$ \\
\hline
\end{tabular} \begin{tabular}{c|cccccc|c} 
f112 & 110 & 1700 & 3400 & 4500 & 5100 & 5600 & $15 / 15$ \\
$(1,2)-C M A-E S$ & 8 & 9.3 & 7 & 7.8 & 7.4 & 7.1 & $12 / 15$ \\
\hline
\end{tabular}

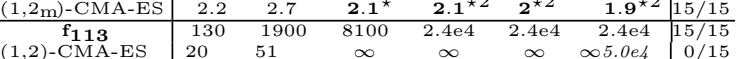
\begin{tabular}{c|rcccccc}
$(1,2 \mathrm{~m})$-CMA-ES & 7.1 & $\mathbf{3 . 1}^{\star 3}$ & $\mathbf{1 3}^{\star 3}$ & $\infty$ & $\infty$ & $\infty 5.0 \mathrm{e} 4$ & $0 / 15$ \\
\hline $\mathbf{f}_{\mathbf{1 1 4}}$ & 770 & $1.5 \mathrm{e} 4$ & $5.6 \mathrm{e} 4$ & $8.3 \mathrm{e} 4$ & $8.3 \mathrm{e} 4$ & $8.5 \mathrm{e} 4$ & $15 / 15$
\end{tabular} \begin{tabular}{c|cccccc|c}
$(1,2)$-CMA-ES & 100 & $\infty$ & $\infty$ & $\infty$ & $\infty$ & $\infty 5.0 e 4$ & $0 / 15$ \\
$(1,2 \mathrm{~m})$-CMA-ES & 25 & $\infty$ & $\infty$ & $\infty$ & $\infty$ & $\infty 5.0 e 4$ & $0 / 15$ \\
\hline $\mathbf{f}_{115}$ & 64 & 490 & 1800 & 2600 & 2600 & 3000 & $15 / 15$ \\
$(1,2)-$ CMA-ES & 5.3 & 5.7 & 66 & $\infty$ & $\infty$ & $\infty 5.0 e 4$ & $0 / 15$
\end{tabular} \begin{tabular}{c|ccccccc}
$(1,2 \mathrm{~m})-\mathrm{CMA}-\mathrm{ES}$ & 1.9 & 2.4 & \multicolumn{2}{c}{$\mathbf{6 . 6}^{\star 2} \mathbf{1 4 0}^{\star}$} & $\mathbf{1 4 0}^{\star}$ & $\mathbf{2 4 0}^{\star}$ & $0 / 15$ \\
\hline $\mathbf{f}_{\mathbf{1 1 6}}$ & 5700 & $1.4 \mathrm{e} 4$ & $2.2 \mathrm{e} 4$ & $2.7 \mathrm{e} 4$ & $3.0 \mathrm{e} 4$ & $3.2 \mathrm{e} 4$ & $15 / 15$
\end{tabular}

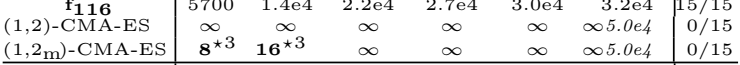
\begin{tabular}{c|cccccc|c}
$(1,2 \mathrm{~m})-\mathrm{CMA}-\mathrm{ES}$ & $\mathbf{8}^{\star 3}$ & $\mathbf{1 6}^{\star 3}$ & $\infty$ & $\infty$ & $\infty$ & $\infty 5.0 \mathrm{e} 4$ & $0 / 15$ \\
\hline $\mathbf{f}_{1 \mathbf{1}}$ & $2.7 \mathrm{e} 4$ & $7.6 \mathrm{e} 4$ & $1.1 \mathrm{e} 5$ & $1.4 \mathrm{e} 5$ & $1.7 \mathrm{e} 5$ & $1.9 \mathrm{e} 5$ & $15 / 15$ \\
& & & & & & $\infty 5.0 \mathrm{e}$ & $0 / 15$
\end{tabular} \begin{tabular}{c|cccccc|c}
$(1,2)-C M A-E S$ & $\infty$ & $\infty$ & $\infty$ & $\infty$ & $\infty$ & $\infty 5.0 e 4$ & $0 / 15$ \\
$(1,2 \mathrm{~m})$-CMA-ES & $\infty$ & $\infty$ & $\infty$ & $\infty$ & $\infty$ & $\infty 5.0 e 4$ & $0 / 15$ \\
\hline $\mathbf{f}_{118}$ & 430 & 1200 & 1600 & 2000 & 2400 & 2900 & $15 / 15$
\end{tabular} \begin{tabular}{c|clllll|l} 
f118 & 430 & 1200 & 1600 & 2000 & 2400 & 2900 & $15 / 15$ \\
$(1,2)-C M A-E S$ & 9.2 & 6.5 & 6.4 & 7.8 & 8.2 & 8.8 & $15 / 15$ \\
$(1,2$ m)-CMA-ES & 4.6 & $\mathbf{2 . 4}^{\star 2}$ & $\mathbf{2 . 2}^{\star 3}$ & $\mathbf{2 . 2}^{\star 3}$ & $\mathbf{2}^{\star 3}$ & $\mathbf{1 . 8}^{\star 3}$ & $15 / 15$ \\
\hline
\end{tabular}

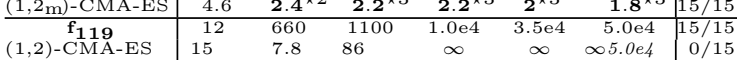
\begin{tabular}{c|cccccr|r}
$(1,2 \mathrm{~m})-\mathrm{CMA}-\mathrm{ES}$ & 6.4 & 2.5 & $\mathbf{6 . 1} \star 2$ & $\infty$ & $\infty$ & $\infty 5.0 \mathrm{e} 4$ & $0 / 15$ \\
\hline $\mathbf{f}_{120}$ & 16 & 2900 & $1.9 \mathrm{e} 4$ & $7.2 \mathrm{e} 4$ & $3.3 \mathrm{e} 5$ & $5.5 \mathrm{e} 5$ & $15 / 15$
\end{tabular} \begin{tabular}{c|cccccc|c}
\hline $\mathbf{f}_{\mathbf{1 2 0}}$ & 16 & 2900 & $1.9 \mathrm{e} 4$ & $7.2 \mathrm{e} 4$ & $3.3 \mathrm{e} 5$ & $5.5 \mathrm{e} 5$ & $15 / 15$ \\
$(1,2)-\mathrm{CMA}-\mathrm{eS}$ & 77 & 33 & $\infty$ & $\infty$ & $\infty$ & $\infty 5.0 e 4$ & $0 / 15$ \\
$(1,2 \mathrm{~m})$-CMA-ES & 46 & 79 & 40 & $\infty$ & $\infty$ & $\infty 5.0 e 4$ & $0 / 15$ \\
\hline
\end{tabular} \begin{tabular}{c|cccccc|r}
$\mathbf{f}_{\mathbf{1 2 1}}$ & 8.6 & 110 & 270 & 1600 & 3900 & 6200 & $15 / 15$ \\
$(1,2)-\mathrm{CMA}-\mathrm{ES}$ & 4.5 & 2.9 & 1.9 & 2.9 & 4.5 & 9 & $4 / 15$
\end{tabular}

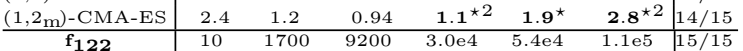
\begin{tabular}{l|cccccc|c}
$(1,2)$-CMA-ES & 11 & 30 & $\infty$ & $\infty$ & $\infty$ & $\infty 5.0 e 4$ & $0 / 15$ \\
\hline
\end{tabular} \begin{tabular}{c|llcccr|r}
$(1,2 \mathrm{~m})$-CMA-ES & 6 & $\mathbf{4 . 7}^{\star 2}$ & $\infty$ & $\infty$ & $\infty$ & $\infty 5.0 e 4$ & $0 / 15$ \\
\hline $\mathbf{f}_{123}$ & 11 & $1.6 \mathrm{e} 4$ & $8.2 \mathrm{e} 4$ & $3.4 \mathrm{e} 5$ & $6.7 \mathrm{e} 5$ & $2.2 \mathrm{e} 6$ & $15 / 15$
\end{tabular} \begin{tabular}{l|llllll|l}
$(1,2)$-CMA-ES & 75 & $\infty$ & $\infty$ & $\infty$ & $\infty$ & $\infty 5.0 e 4$ & $0 / 15$ \\
$(1,2 \mathrm{~m})$-CMA-ES & 61 & 44 & $\infty$ & $\infty$ & $\infty$ & $\infty 5.0 e 4$ & $0 / 15$ \\
\hline
\end{tabular} \begin{tabular}{c|cccccc|r}
$\mathbf{f}_{\mathbf{1 2 4}}$ & 9.7 & 200 & 1000 & $2.0 \mathrm{e} 4$ & $4.5 \mathrm{e} 4$ & $9.5 \mathrm{e} 4$ & $15 / 15$ \\
$(1,2)-\mathrm{CMA}-\mathrm{ES}$ & 11 & 130 & 700 & $\infty$ & $\infty$ & $\infty 5.0 e 4$ & $0 / 15$
\end{tabular} \begin{tabular}{c|cccccc|c}
$(1,2 \mathrm{~m})-\mathrm{CMA}-\mathrm{ES}$ & 3.4 & $\mathbf{2 . 7}^{\star 3}$ & $\mathbf{3 0} \mathbf{0}^{\star 3}$ & $\infty$ & $\infty$ & $\infty 5.0 e 4$ & $0 / 15$ \\
\hline $\mathrm{f}_{125}$ & 1 & 1 & 1 & $2.4 \mathrm{e} 5$ & $2.4 \mathrm{e} 5$ & $2.5 \mathrm{e} 5$ & $15 / 15$
\end{tabular} \begin{tabular}{l|lrrrrr|r}
$(1,2)-$ CMA-ES & 1 & 120 & $5.9 \mathrm{e} 4$ & $\infty$ & $\infty$ & $\infty 5.0 e 4$ & $0 / 15$ \\
$(1,2 \mathrm{~m})$-CMA-ES & 1.3 & 69 & $1.5 \mathrm{e} 4$ & $\infty$ & $\infty$ & $\infty 5.0 e 4$ & $0 / 15$ \\
\hline
\end{tabular} \begin{tabular}{c|cccccc|l}
$\mathbf{f}_{126}$ & 1 & 1 & 1 & $\infty$ & $\infty$ & $\infty$ & 0 \\
$(1,2)$-CMA-ES & 1.1 & $2.0 \mathrm{e} 3$ & $7.4 \mathrm{e} 5$ & $\infty$ & $\infty$ & $\infty$ & $0 / 15$
\end{tabular} \begin{tabular}{c|cccccc|c}
$(1,2 \mathrm{~m})$-CMA-ES & 1 & 620 & $\infty$ & $\infty$ & $\infty$ & $\infty$ & $0 / 15$ \\
\hline$f_{127}$ & 1 & 1 & 1 & $3.4 \mathrm{e} 5$ & $3.9 \mathrm{e} 5$ & $4.0 \mathrm{e} 5$ & $15 / 15$
\end{tabular} \begin{tabular}{l|cccccc|c}
$(1,2)$-CMA-ES & 1 & 85 & $1.4 \mathrm{e} 4$ & $\infty$ & $\infty$ & $\infty 5.0 e 4$ & $0 / 15$ \\
\hline
\end{tabular} \begin{tabular}{c|cccccr|r}
$(1,2 \mathrm{~m})-\mathrm{CMA}-\mathrm{ES}$ & 1 & 31 & $5.2 \mathrm{e} 3$ & $\infty$ & $\infty$ & $\infty 5.0 \mathrm{e} 4$ & $0 / 15$ \\
\hline $\mathbf{f}_{128}$ & 110 & 4200 & 7800 & $1.2 \mathrm{e} 4$ & $1.7 \mathrm{e} 4$ & $2.1 \mathrm{e} 4$ & $15 / 15$
\end{tabular}

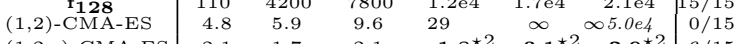

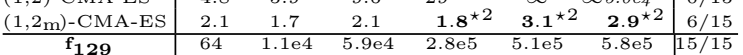
\begin{tabular}{l|lllllll}
$(1,2)$-CMA-ES & 170 & $\infty$ & $\infty$ & $\infty$ & $\infty$ & $\infty 5.0 e 4$ & $0 / 15$ \\
$(1,2 \mathrm{~m})$-CMA-ES & 110 & $\infty$ & $\infty$ & $\infty$ & $\infty$ & $\infty 5.0 e 4$ & $0 / 15$ \\
\hline
\end{tabular} \begin{tabular}{c|cccccc|r} 
f $_{130}$ & 55 & 810 & 3000 & $3.3 \mathrm{e} 4$ & $3.4 \mathrm{e} 4$ & $3.5 \mathrm{e} 4$ & $10 / 15$ \\
$(1,2)-C M A-E S$ & 14 & 48 & 23 & 2.9 & 2.8 & 3.6 & $5 / 15$ \\
& 7.2 & 17 & 8.7 & 0.82 & 0.8 & 0.79 &
\end{tabular}
20-D

\begin{tabular}{|c|c|c|c|c|c|c|c|}
\hline$\Delta f$ & $1 \mathrm{e}+1$ & $1 \mathrm{e}+0$ & $1 e-1$ & $1 e-3$ & $1 e-5$ & $1 e-7$ & \#succ \\
\hline$f_{101}$ & 59 & 360 & 510 & 700 & 740 & 780 & $15 / 15$ \\
\hline ,2)-CMA-1 & 17 & 4 & 3.7 & 4 & 5 & 5.8 & $15 / 15$ \\
\hline n)-CMA- & $9.1^{\star 3}$ & $2.2^{\star 3}$ & $2^{\star 3}$ & $2.1^{\star 3}$ & $2.7^{\star 3}$ & $3.1 \star 3$ & $15 / 15$ \\
\hline$f_{102}$ & 230 & 400 & 580 & 920 & 1200 & 1400 & $15 / 15$ \\
\hline$-\mathrm{CMA}-1$ & 5 & 4.1 & 3.7 & 4.1 & 5.3 & 9.8 & \\
\hline m)-CMA-ES & $2.5^{\star 3}$ & $2^{\star 3}$ & $1.8^{\star 3}$ & $1.7^{\star 3}$ & $1.7^{\star 3}$ & $1.8^{\star 3}$ & $15 / 15$ \\
\hline$f_{103}$ & 65 & 420 & 630 & 1300 & 1900 & 2500 & $14 / 15$ \\
\hline ,2)-CMA-E & 18 & 3.7 & 3.1 & 2.2 & 2.1 & $2^{2}$ & $15 / 15$ \\
\hline $2 \mathrm{~m})$-CMA-ES & $7.7^{\star 3}$ & $1.7^{\star 3}$ & $1.6^{\star 3}$ & $1.1 \star 3$ & $1.1^{\star 3}$ & $1.1 \star 3$ & $15 / 15$ \\
\hline & $2.4 \mathrm{e} 4$ & $8.6 \mathrm{e} 4$ & $1.7 \mathrm{e} 5$ & $1.8 \mathrm{e} 5$ & $1.9 \mathrm{e} 5$ & $2.0 \mathrm{e} 5$ & $15 / 15$ \\
\hline$-\mathrm{CMA}-\mathrm{E}$ & 63 & $\infty$ & $\infty$ & $\infty$ & $\infty$ & $\infty 2.0 e 5$ & $0 / 15$ \\
\hline n)-CMA- & 28 & 34 & $\infty$ & $\infty$ & $\infty$ & $\infty 2.0 e 5$ & $0 / 15$ \\
\hline$f_{105}$ & $1.9 \mathrm{e} 5$ & $6.1 \mathrm{e} 5$ & $6.3 \mathrm{e} 5$ & $6.5 \mathrm{e} 5$ & $6.6 \mathrm{e} 5$ & $6.7 \mathrm{e} 5$ & $15 / 15$ \\
\hline -CMA-ES & & 4.9 & $\infty$ & $\infty$ & $\infty$ & $\infty 2.0 e 5$ & \\
\hline ,2m)-CMA-ES & 2.7 & $\infty$ & $\infty$ & $\infty$ & $\infty$ & $\infty 2.005$ & $0 / 15$ \\
\hline$f_{106}$ & $1.1 \mathrm{e} 4$ & $2.2 \mathrm{e} 4$ & $2.4 \mathrm{e} 4$ & $2.5 \mathrm{e} 4$ & $2.6 \mathrm{e} 4$ & $2.7 \mathrm{e} 4$ & \\
\hline -CMA & 2.3 & 3.9 & 3.9 & 4.1 & 4.1 & & $14 / 15$ \\
\hline, $2 \mathrm{~m})$-CMA-ES & $0.88^{\star}$ & $1.2^{\star 3}$ & $1.3^{\star 3}$ & $1.3^{\star 3}$ & $1.3^{\star 3}$ & $1.3^{\star 3}$ & $15 / 15$ \\
\hline$f_{107}$ & 8600 & $1.4 \mathrm{e} 4$ & $1.6 \mathrm{e} 4$ & $2.7 \mathrm{e} 4$ & $5.2 \mathrm{e} 4$ & $6.5 \mathrm{e} 4$ & $15 / 15$ \\
\hline $\mathrm{CMA}$ & $\infty$ & $\infty$ & $\infty$ & $\infty$ & $\infty$ & $\infty 2.0 e 5$ & $0 / 15$ \\
\hline m)-CMA & $\infty$ & $\infty$ & $\infty$ & $\infty$ & $\infty$ & $02.0 e 5$ & \\
\hline$f_{108}$ & $5.8 \mathrm{e} 4$ & $9.7 \mathrm{e} 4$ & $2.0 \mathrm{e} 5$ & $4.5 \mathrm{e} 5$ & $6.3 \mathrm{e} 5$ & $9.0 \mathrm{e} 5$ & $15 / 15$ \\
\hline$-\mathrm{CMA}$ & $\infty$ & $\infty$ & $\infty$ & $\infty$ & $\infty$ & $\infty 2.0 e 5$ & $0 / 15$ \\
\hline m) $-\mathrm{CM}$ & $\infty$ & $\infty$ & $\infty$ & $\infty$ & $\infty$ & $\infty 2.0 e 5$ & \\
\hline$f_{109}$ & 330 & 630 & 1100 & 2300 & 3600 & 5000 & 15 \\
\hline - $-\mathrm{CMA}-\mathrm{E}$ & 3.6 & 3.5 & 2.7 & 2.4 & 2.3 & 2.2 & \\
\hline, $2 \mathrm{~m})$-CMA-ES & $1.7 \star 3$ & $1.5^{\star 3}$ & $1.4^{\star 3}$ & $1.1^{\star 3}$ & $1^{\star 3}$ & $0.96^{\star 3}$ & $15 / 15$ \\
\hline$f_{110}$ & $\infty$ & $\infty$ & $\infty$ & $\infty$ & $\infty$ & $\infty$ & \\
\hline & $\infty$ & $\alpha$ & $\infty$ & $\infty$ & & $\infty$ & $/ 15$ \\
\hline n)-CM & $\infty$ & $\infty$ & $\infty$ & $\infty$ & $\infty$ & $\infty$ & $0 / 15$ \\
\hline$f_{111}$ & $\infty$ & $\infty$ & $\infty$ & $\infty$ & $\infty$ & $\infty$ & 0 \\
\hline & & & $\infty$ & $\infty$ & & $\infty$ & $0 / 15$ \\
\hline n)-CM & $\infty$ & $\infty$ & $\infty$ & $\infty$ & $\infty$ & $\infty$ & $0 / 15$ \\
\hline$f_{112}$ & $2.6 \mathrm{e} 4$ & $6.4 \mathrm{e} 4$ & $7.0 \mathrm{e} 4$ & $7.4 \mathrm{e} 4$ & $7.6 \mathrm{e} 4$ & $7.8 \mathrm{e} 4$ & $15 / 15$ \\
\hline -CMA-ES & 3.1 & 8.4 & & 40 & 39 & & $1 / 15$ \\
\hline m)-CMA- & $0.98^{\star 3}$ & $1.2^{\star 2}$ & $1.2^{\star 3}$ & $1.2^{\star 3}$ & $1.3^{\star 3}$ & $1.3^{\star 3}$ & $15 / 15$ \\
\hline$f_{113}$ & $5.0 \mathrm{e} 4$ & $3.6 \mathrm{e} 5$ & $5.6 \mathrm{e} 5$ & $5.9 \mathrm{e} 5$ & $5.9 \mathrm{e} 5$ & $5.9 \mathrm{e} 5$ & $15 / 15$ \\
\hline IIS & $\infty$ & $\infty$ & $\infty$ & $\infty$ & $\infty$ & $\infty 2$. & \\
\hline ) $-\mathrm{CM}$ & $\infty$ & $\infty$ & $\infty$ & $\infty$ & $\infty$ & $\infty 2.0 e 5$ & \\
\hline$f_{114}$ & $2.1 \mathrm{e} 5$ & $1.1 \mathrm{e} 6$ & $1.4 \mathrm{e} 6$ & $1.6 \mathrm{e} 6$ & $1.6 \mathrm{e} 6$ & $1.6 \mathrm{e} 6$ & $15 / 15$ \\
\hline 114 & $\infty$ & $\infty$ & $\infty$ & $\infty$ & $\infty$ & $\infty 2$. & \\
\hline )- $-C M$ & $\infty$ & $\infty$ & $\infty$ & $\infty$ & $\infty$ & $\infty 2.6$ & \\
\hline$f_{115}$ & 2400 & $3.0 \mathrm{e} 4$ & $9.2 \mathrm{e} 4$ & $1.3 \mathrm{e} 5$ & $1.3 \mathrm{e} 5$ & $1.3 \mathrm{e} 5$ & $15 / 15$ \\
\hline )-CMA- & 1.2 & $\infty$ & $\infty$ & $\infty$ & $\infty$ & $\infty 2.6$ & $0 / 15$ \\
\hline $2 \mathrm{~m})$-CMA-ES & $7^{\star 3}$ & $\infty$ & $\infty$ & $\infty$ & $\infty$ & $\infty 2.6$ & $0 / 15$ \\
\hline$f_{116}$ & $5.0 \mathrm{e} 5$ & $6.9 \mathrm{e} 5$ & $8.9 \mathrm{e} 5$ & $1.0 \mathrm{e} 6$ & $1.1 \mathrm{e} 6$ & & $15 / 15$ \\
\hline$-\mathrm{CMA}$ & $\infty$ & $\infty$ & $\infty$ & $\infty$ & $\infty$ & $\infty 2.0$ & $0 / 15$ \\
\hline m) $-\mathrm{CM}$ & $\infty$ & $\infty$ & $\infty$ & $\infty$ & $\infty$ & $\infty 2.0 e 5$ & $0 / 15$ \\
\hline$f_{117}$ & $1.8 \mathrm{e} 6$ & $2.5 \mathrm{e} 6$ & $2.6 \mathrm{e} 6$ & $2.9 \mathrm{e} 6$ & $3.2 \mathrm{e} 6$ & & $15 / 15$ \\
\hline )-CMA & $\infty$ & $\infty$ & $\infty$ & $\infty$ & $\infty$ & $\infty 2.0 e 5$ & $0 / 15$ \\
\hline m)-CM & $\infty$ & $\infty$ & $\infty$ & $\infty$ & $\infty$ & $\infty 2.0 e 5$ & \\
\hline$f_{118}$ & 6900 & $1.2 \mathrm{e} 4$ & $1.8 \mathrm{e} 4$ & $2.6 \mathrm{e} 4$ & $3.0 \mathrm{e} 4$ & & $15 / 15$ \\
\hline ,2)-CMA- & 4.6 & 10 & 20 & & 98 & $\infty 2.0 e 5$ & $0 / 15$ \\
\hline, $2 \mathrm{~m})$-CMA-ES & $1.7^{\star 3}$ & $1.7 \star 3$ & $1.5^{\star 3}$ & $1.5^{\star 3}$ & $1.5^{\star 3}$ & $1.4^{\star 3}$ & $15 / 15$ \\
\hline$f_{119}$ & 2800 & $2.9 \mathrm{e} 4$ & $3.6 \mathrm{e} 4$ & $4.1 \mathrm{e} 5$ & $1.4 \mathrm{e} 6$ & $1.9 \mathrm{e} 6$ & $15 / 15$ \\
\hline - CMA & 200 & $\infty$ & $\infty$ & $\infty$ & $\infty$ & $\infty 2.0 e 5$ & $0 / 15$ \\
\hline $2 \mathrm{~m})$-CMA-ES & $520^{\star 2}$ & $\infty$ & $\infty$ & $\infty$ & $\infty$ & $\infty 2.0 e 5$ & $0 / 15$ \\
\hline$f_{120}$ & $3.6 \mathrm{e} 4$ & $1.8 \mathrm{e} 5$ & $2.8 \mathrm{e} 5$ & $1.6 \mathrm{e} 6$ & $6.7 \mathrm{e} 6$ & & \\
\hline$-\mathrm{CMA}$ & $\infty$ & $\infty$ & $\infty$ & $\infty$ & $\infty$ & $\infty 2.0 e 5$ & $0 / 15$ \\
\hline n) $-\mathrm{CM}$ & $\infty$ & $\infty$ & $\infty$ & $\infty$ & $\infty$ & $\infty 2$ & \\
\hline$f_{121}$ & 250 & 770 & 1400 & 9300 & $3.4 \mathrm{e} 4$ & 5.7 & $15 / 15$ \\
\hline - CMA & 4.8 & 3.4 & 3 & 1.9 & 3.8 & $\infty 2.0 \mathrm{e} 5$ & $0 / 15$ \\
\hline, $2 \mathrm{~m})$-CMA-ES & $2.2^{\star 2}$ & $1.4^{\star 3}$ & $1.2^{\star 3}$ & $0.81^{\star 3 \downarrow 2}$ & $0.8^{\star 3 \downarrow}$ & $6.2^{\star 3}$ & $0 / 15$ \\
\hline$f_{122}$ & 690 & $5.2 \mathrm{e} 4$ & $1.4 \mathrm{e} 5$ & $7.9 \mathrm{e} 5$ & $2.0 \mathrm{e} 6$ & $5.8 \mathrm{e} 6$ & $15 / 15$ \\
\hline 122 & 99 & $\infty$ & $\infty$ & $\infty$ & $\infty$ & & \\
\hline m)-CM & 45 & $\infty$ & $\infty$ & $\infty$ & $\infty$ & $\infty 2$ & $0 / 15$ \\
\hline$f_{123}$ & 110 & $5.3 \mathrm{e} 5$ & $1.5 \mathrm{e} 6$ & $5.3 \mathrm{e} 6$ & $2.7 \mathrm{e} 7$ & & \\
\hline & & $\propto$ & $\propto$ & $\infty$ & $\alpha$ & $\infty 2$ & \\
\hline a)-CM & 0 & $\alpha$ & $\propto$ & $\alpha$ & & $\infty 2$. & \\
\hline$f_{124}$ & 19 & 2000 & $4.1 \mathrm{e} 4$ & $1.3 \mathrm{e} 5$ & $3.9 \mathrm{e} 5$ & & \\
\hline & & $\infty$ & $\infty$ & $\infty$ & $\infty$ & $\infty 2$ & $0 / 15$ \\
\hline ) $-\mathrm{CM}$ & $3.7^{\star}$ & $\infty$ & $\infty$ & $\infty$ & $\infty$ & $\infty 2$. & \\
\hline$f_{125}$ & 1 & 1 & 1 & $2.5 \mathrm{e} 7$ & $8.0 \mathrm{e} 7$ & & $\frac{47}{4 /}$ \\
\hline CMA & 1 & & $\infty$ & $\infty$ & $\alpha$ & & $0 / 15$ \\
\hline ) $-\mathrm{CM}$ & 1 & $5 e^{5}$ & $\infty$ & $\infty$ & $\infty$ & $\infty 2.0 e 5$ & $0 / 15$ \\
\hline$f_{126}$ & 1 & 1 & 1 & $\infty$ & & $\infty$ & \\
\hline$-C N 1 A$ & 1 & & & & & & \\
\hline m)-CM & 1 & $\infty$ & $\infty$ & $\infty$ & & $\infty$ & $0 / 15$ \\
\hline$f_{127}$ & 1 & 1 & 1 & $4.4 \mathrm{e} 6$ & $7.3 \mathrm{e} 6$ & $7.4 \mathrm{e} 6$ & $15 / 15$ \\
\hline $\mathrm{CMA}$ & 1 & & $\infty$ & $\infty$ & $\infty$ & & $0 / 15$ \\
\hline $2 \mathrm{~m})-\mathrm{CMA}$ & 1 & $600^{\star 3}$ & $\infty$ & $\infty$ & $\infty$ & $\infty 2$. & $0 / 15$ \\
\hline$f_{128}$ & $1.4 \mathrm{e}$ & $1.3 \mathrm{e} 7$ & $1.7 \mathrm{e} 7$ & $1.7 \mathrm{e} 7$ & $1.7 \mathrm{e} 7$ & 1.7 & $9 / 15$ \\
\hline & $\propto$ & $\infty$ & $\infty$ & $\infty$ & $\infty$ & $\infty 2$. & $0 / 15$ \\
\hline )-CM & & & & $\infty$ & & $\infty 2$ & \\
\hline$f_{129}$ & $7.8 \mathrm{e}$ & $4.1 \mathrm{e} 7$ & $4.2 \mathrm{e} 7$ & $4.2 \mathrm{e} 7$ & $4.2 \mathrm{e} 7$ & $4.2 \mathrm{e} 7$ & $5 / 15$ \\
\hline & & $\infty$ & $\infty$ & . & & & \\
\hline n)-CMA & & & & $\infty$ & & $\infty 2$ & \\
\hline & 490 & $9.3 \mathrm{e} 4$ & $2.5 \mathrm{e} 5$ & $2.5 \mathrm{e} 5$ & $2.6 \mathrm{e} 5$ & $2.6 \mathrm{e} 5$ & $7 / 15$ \\
\hline & 2. & & & & & 0.97 & \\
\hline CM & 1 & 0.61 & 0.61 & 0.61 & 0.61 & 0.61 & $10 / 15$ \\
\hline
\end{tabular}

Table 1: ERT in number of function evaluations divided by the best ERT measured during BBOB-2009 (given in the respective first row) for the algorithms $(1,2)$-CMA-ES and $\left(1,2_{\mathrm{m}}\right)$-CMA-ES for different $\Delta f$ values for functions $f_{101}-f_{130}$. The median number of conducted function evaluations is additionally given in italics, if $\operatorname{ERT}\left(10^{-7}\right)=\infty$. \#succ is the number of trials that reached the final target $f_{\mathrm{opt}}+10^{-8}$. Bold entries are statistically significantly better compared to the other algorithm, with $p=0.05$ or $p=10^{-k}$ where $k>1$ is the number following the $\star$ symbol, with Bonferroni correction of 60 . 
101 Sphere (moderate) 104 Rosenbrock (moderate)

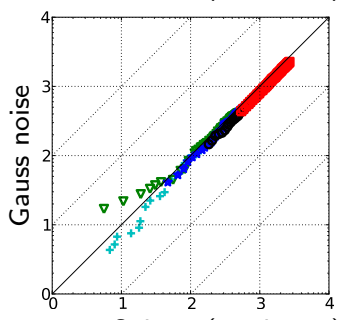

102 Sphere (moderate)

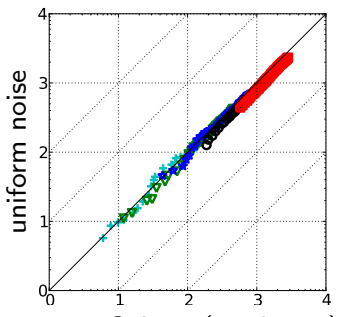

103 Sphere (moderate)

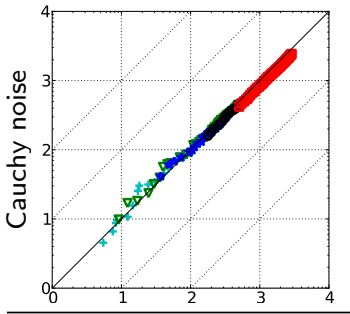

116 Ellipsoid

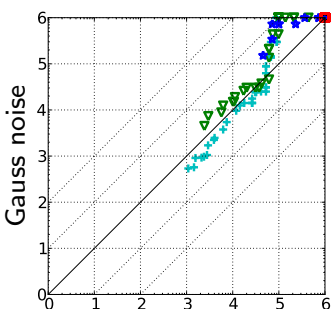

117 Ellipsoid

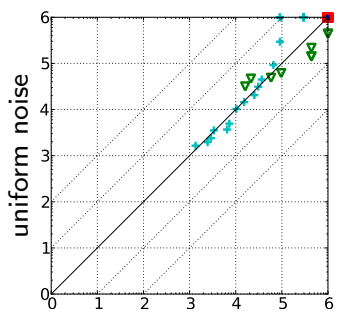

118 Ellipsoid

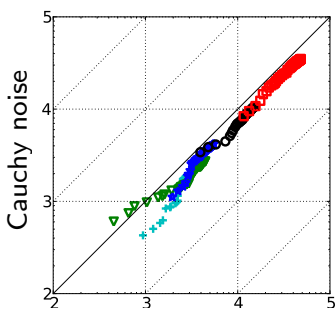

107 Sphere

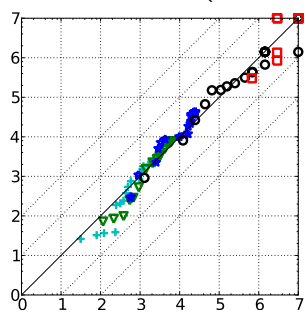

105 Rosenbrock (moderate)

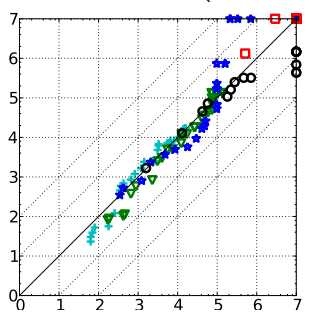

106 Rosenbrock (moderate)
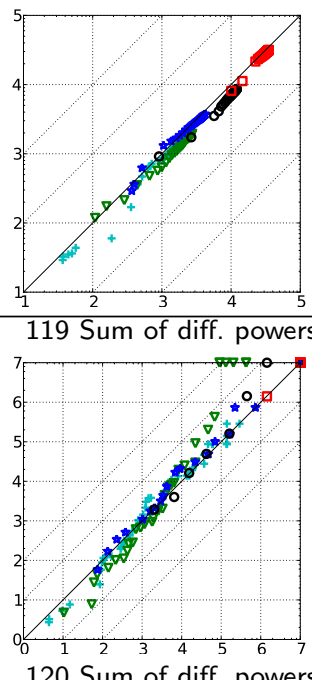

120 Sum of diff. powers

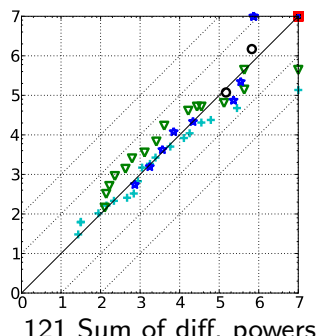

121 Sum of diff. powers

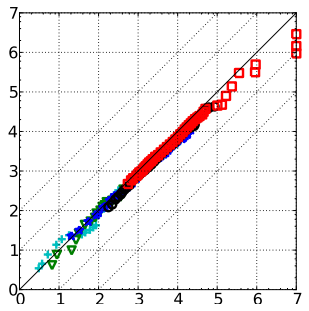

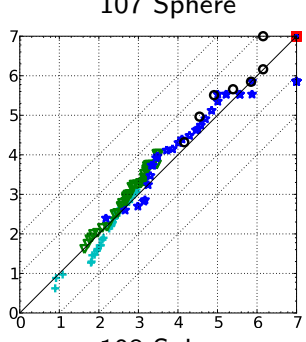

108 Sphere

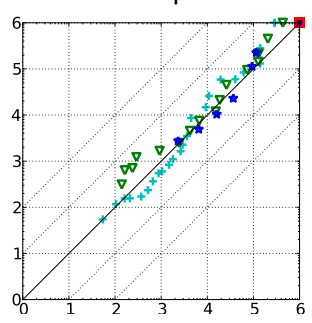

109 Sphere

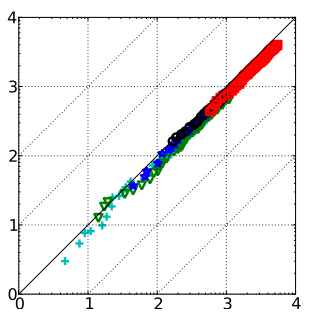

122 Schaffer F7

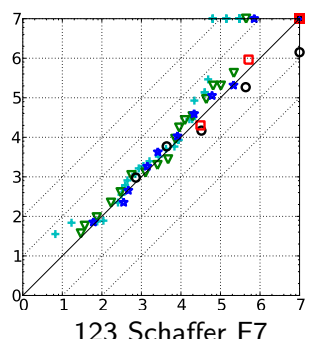

123 Schaffer F7

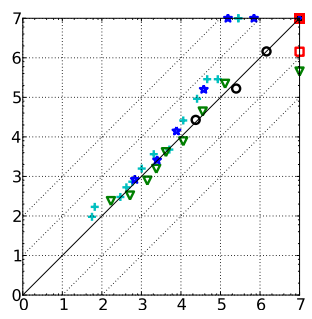

124 Schaffer F7

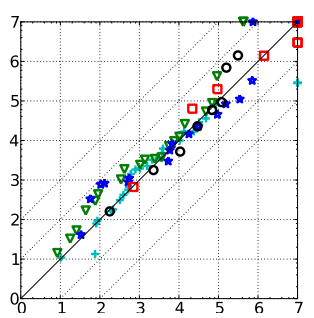

110 Rosenbrock

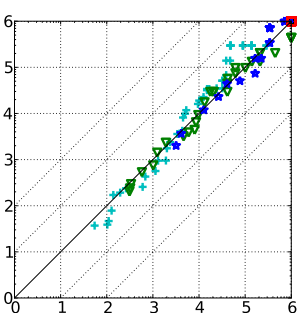

111 Rosenbrock

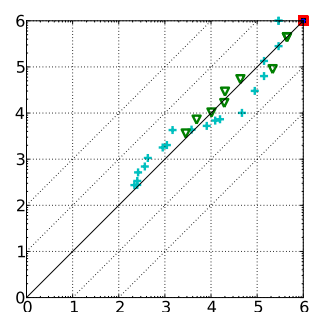

112 Rosenbrock

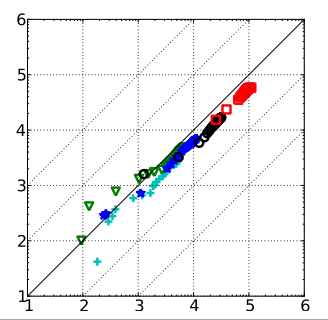

125 Griewank-Rosenbrock

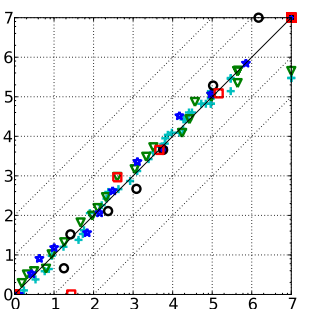

126 Griewank-Rosenbrock

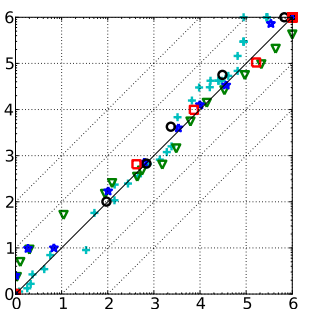

127 Griewank-Rosenbrock

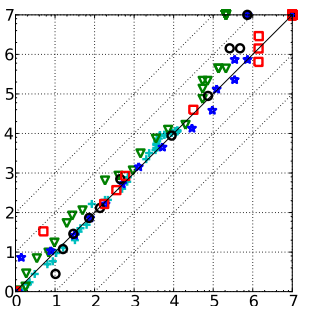

113 Step-ellipsoid

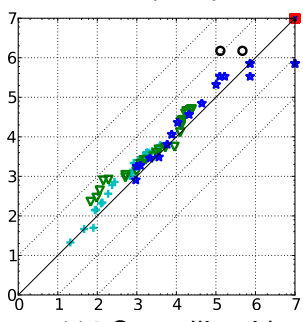

114 Step-ellipsoid

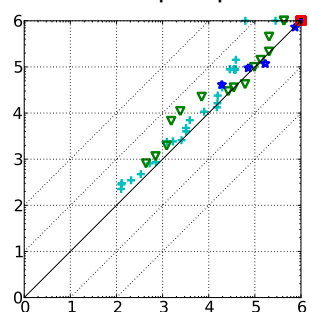

115 Step-ellipsoid

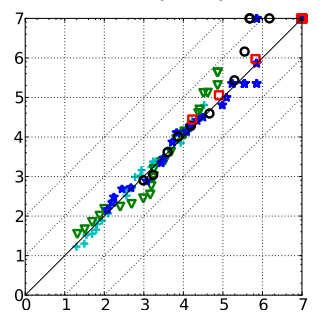

128 Gallagher

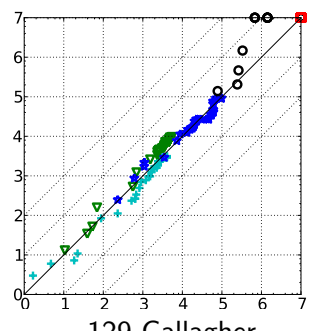

129 Gallagher

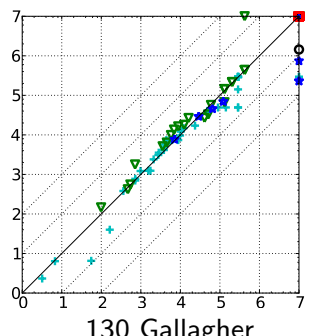

130 Gallagher

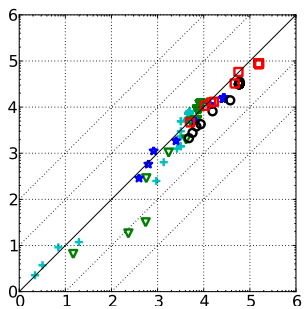

Figure 3: Expected running time (ERT in log10 of number of function evaluations) of (1, $2_{\mathrm{m}}^{\mathrm{s}}$ )-CMA-ES versus $\left(1,2_{\mathrm{m}}\right)$-CMA-ES for 46 target values $\Delta f \in\left[10^{-8}, 10\right]$ in each dimension for functions $f_{101}-f_{130}$. Markers on the upper or right edge indicate that the target value was never reached by $\left(1,2_{\mathrm{m}}^{\mathrm{s}}\right)-\mathrm{CMA}-\mathrm{ES}$ or $\left(1,2_{\mathrm{m}}\right)-\mathrm{CMA}-\mathrm{ES}$ respectively. Markers represent dimension: 2: $+, 3: \nabla, 5: \star, 10: \circ, 20: \square$. 
5-D

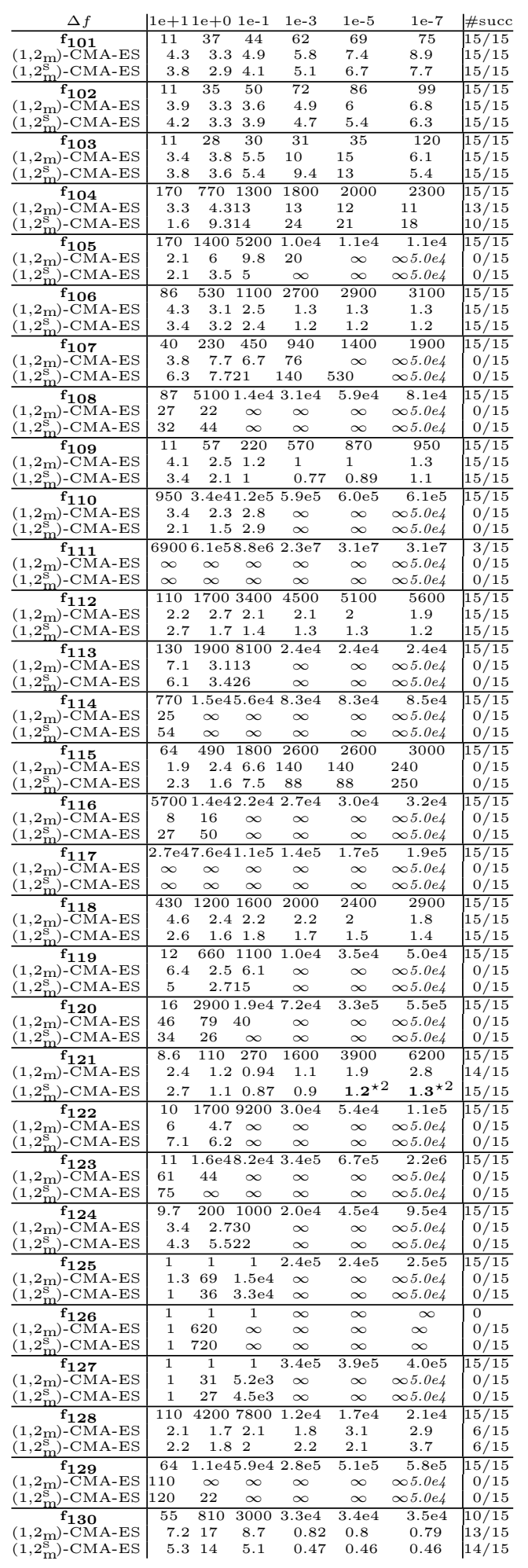

20-D

\begin{tabular}{|c|c|c|c|c|c|c|c|}
\hline$\Delta f$ & $1 \mathrm{e}+1$ & $1 \mathrm{e}+0$ & $1 e-1$ & $1 \mathrm{e}-3$ & $1 e-5$ & $1 \mathrm{e}-7$ & \#succ \\
\hline$f_{101}$ & 59 & 360 & 510 & 700 & 740 & 780 & $15 / 15$ \\
\hline$(1,2 \mathrm{~m})-\mathrm{CMA}-$ & 9.1 & 2.2 & 2 & 2.1 & 2.7 & 3.1 & $15 / 15$ \\
\hline$\left(1,2_{\mathrm{m}}^{\mathrm{s}}\right)-\mathrm{CMA}-\mathrm{ES}$ & 7.4 & $1.8^{\star}$ & $1.7^{\star 2}$ & $1.8^{\star 3}$ & $2.2^{\star 3}$ & $2.6^{\star 3}$ & $15 / 15$ \\
\hline$f_{102}$ & 230 & 400 & 580 & 920 & 1200 & 1400 & $15 / 15$ \\
\hline 2m)-CMA- & 2.5 & 2 & 1.8 & 1.7 & 1.7 & 1.8 & $15 / 15$ \\
\hline )-CMA-ES & $1.9^{\star 2}$ & $1.6^{\star 2}$ & $1.4^{\star 2}$ & $1.3^{\star 2}$ & $1.5^{\star 2}$ & $1.5^{\star 3}$ & $15 / 15$ \\
\hline$f_{103}$ & 65 & 420 & 630 & 1300 & 1900 & 2500 & $14 / 15$ \\
\hline m)-CMA- & 7.7 & 1.7 & 1.6 & 1.1 & 1.1 & 1.1 & $15 / 15$ \\
\hline$(1,2 \mathrm{~m})-\mathrm{CMA}-\mathrm{ES}$ & 6.4 & 1.5 & 1.3 & $0.95^{\star 2}$ & $0.89^{\star 3 \downarrow}$ & ${ }^{2} 0.88^{\star 3 \downarrow 2}$ & $15 / 15$ \\
\hline$f_{104}$ & $2.4 \mathrm{e} 4$ & $8.6 \mathrm{e} 4$ & $1.7 \mathrm{e} 5$ & $1.8 \mathrm{e} 5$ & $1.9 \mathrm{e} 5$ & $2.0 \mathrm{e} 5$ & $15 / 15$ \\
\hline MA-ES & 28 & 34 & $\infty$ & $\infty$ & $\infty$ & $\infty 2.0 e 5$ & $0 / 15$ \\
\hline )-CMA & 13 & $\infty$ & $\infty$ & $\infty$ & $\infty$ & $\infty 2.0 e 5$ & $0 / 15$ \\
\hline$f_{105}$ & $1.9 \mathrm{e} 5$ & $6.1 \mathrm{e} 5$ & $6.3 \mathrm{e} 5$ & $6.5 \mathrm{e} 5$ & $6.6 \mathrm{e} 5$ & $6.7 \mathrm{e} 5$ & $15 / 15$ \\
\hline $1,2 \mathrm{~m})-\mathrm{C}$ & 2.7 & $\infty$ & $\infty$ & $\infty$ & $\infty$ & $\infty 2.0 e 5$ & $0 / 15$ \\
\hline ( & 6.9 & $\infty$ & $\infty$ & $\infty$ & $\infty$ & $\infty 2.0 e 5$ & $0 / 15$ \\
\hline & $1.1 \mathrm{e} 4$ & $2.2 \mathrm{e} 4$ & $2.4 \mathrm{e} 4$ & $2.5 \mathrm{e} 4$ & $2.6 \mathrm{e} 4$ & $2.7 \mathrm{e} 4$ & $15 / 15$ \\
\hline $1,2 \mathrm{~m})-($ & 0.88 & 1.2 & 1.3 & 1.3 & 1.3 & 1.3 & \\
\hline $2 \mathrm{~m}$ )-CMA-ES & 0.71 & 1.2 & 1.2 & 1.2 & 1.2 & 1.1 & $15 / 15$ \\
\hline$f_{107}$ & 8600 & $1.4 \mathrm{e} 4$ & $1.6 \mathrm{e} 4$ & $2.7 \mathrm{e} 4$ & $5.2 \mathrm{e} 4$ & $6.5 \mathrm{e} 4$ & $15 / 15$ \\
\hline n) $-\mathrm{CMA}$ & $\infty$ & $\infty$ & $\infty$ & $\infty$ & $\infty$ & $\infty 2.0 e 5$ & \\
\hline ()-CMA- & $\infty$ & $\infty$ & $\infty$ & $\infty$ & $\infty$ & $\infty 2.0 e 5$ & $0 / 15$ \\
\hline$f_{108}$ & $5.8 \mathrm{e} 4$ & $9.7 \mathrm{e} 4$ & $2.0 \mathrm{e} 5$ & $4.5 \mathrm{e} 5$ & $6.3 \mathrm{e} 5$ & $9.0 \mathrm{e} 5$ & $15 / 15$ \\
\hline 00 & $\infty$ & $\infty$ & $\infty$ & $\infty$ & $\infty$ & $\infty 2.0 e 5$ & \\
\hline )-CMA & $\infty$ & $\infty$ & $\infty$ & $\infty$ & $\infty$ & $\infty 2.0 e 5$ & $/ 15$ \\
\hline$f_{109}$ & 330 & 630 & 1100 & 2300 & 3600 & 5000 & $15 / 15$ \\
\hline $1,2 \mathrm{~m}$-CMA-ES & 1.7 & 1.5 & 1.4 & 1.1 & 8000 & 0.96 & $15 / 15$ \\
\hline $\left.2_{\mathrm{m}}^{\mathrm{s}}\right)$-CMA-ES & 1.3 & 1.2 & $0.97 \star 3$ & $0.84^{\star 3 \downarrow}$ & $0.77^{\star 3}$ & ${ }^{3} 0.72^{\star 3}$ & $15 / 15$ \\
\hline$f_{110}$ & $\infty$ & $\infty$ & $\infty$ & $\infty$ & $\infty$ & $\infty$ & \\
\hline & $\infty$ & $\infty$ & $\infty$ & $\infty$ & $\infty$ & $\infty$ & \\
\hline )-CMA- & $\infty$ & $\infty$ & $\infty$ & $\infty$ & $\infty$ & $\infty$ & $0 / 15$ \\
\hline & $\infty$ & $\infty$ & $\infty$ & $\infty$ & $\infty$ & $\infty$ & 0 \\
\hline n) $-\mathrm{CMA}$ & $\infty$ & & $\infty$ & $\infty$ & $\infty$ & $\infty$ & $/ 15$ \\
\hline )-CMA-ES & $\infty$ & $\infty$ & $\infty$ & $\infty$ & $\infty$ & $\infty$ & $/ 15$ \\
\hline$f_{112}$ & $2.6 \mathrm{e} 4$ & $6.4 \mathrm{e} 4$ & $7.0 \mathrm{e} 4$ & $7.4 \mathrm{e} 4$ & $7.6 \mathrm{e} 4$ & $7.8 \mathrm{e} 4$ & $15 / 15$ \\
\hline a)-CMA-ES & 0.98 & 1.2 & 1.2 & 1.2 & 1.3 & 1.3 & $15 / 15$ \\
\hline -CMA-ES & $0.6^{\star \downarrow 2}$ & $0.68^{\star \downarrow}$ & $\downarrow 0.71^{\star \downarrow}$ & $0.73^{\star 2 \downarrow}$ & $0.73^{\star 2}$ & $2 \mathbf{0 . 7 3 ^ { \star 2 \downarrow 2 }}$ & $2 \mid 15 / 15$ \\
\hline & $5.0 \mathrm{e} 4$ & $3.6 \mathrm{e} 5$ & $5.6 \mathrm{e} 5$ & $5.9 \mathrm{e} 5$ & $5.9 \mathrm{e} 5$ & $5.9 \mathrm{e} 5$ & $15 / 15$ \\
\hline & $\infty$ & $\infty$ & $\infty$ & $\infty$ & $\infty$ & $\infty 2.0 e 5$ & \\
\hline )-CMA- & $\infty$ & $\infty$ & $\infty$ & $\infty$ & $\infty$ & $\infty 2.0 e 5$ & $/ 15$ \\
\hline$f_{114}$ & $2.1 \mathrm{e} 5$ & $1.1 \mathrm{e} 6$ & $1.4 \mathrm{e} 6$ & $1.6 \mathrm{e} 6$ & $1.6 \mathrm{e} 6$ & $1.6 \mathrm{e} 6$ & $15 / 15$ \\
\hline 104 & $\infty$ & $\infty$ & $\infty$ & $\infty$ & $\infty$ & $\infty 2.0 e 5$ & \\
\hline is & $\infty$ & $\infty$ & $\infty$ & $\infty$ & $\infty$ & $\infty 2.0 e 5$ & 15 \\
\hline & 2400 & $3.0 \mathrm{e} 4$ & $9.2 \mathrm{e} 4$ & $1.3 \mathrm{e} 5$ & $1.3 \mathrm{e} 5$ & $1.3 \mathrm{e} 5$ & $15 / 15$ \\
\hline )- $-\mathrm{CMA}$ & 7 & $\infty$ & $\infty$ & $\infty$ & $\infty$ & $\infty 2$. & \\
\hline )-CMA- & 11 & $\infty$ & $\infty$ & $\infty$ & $\infty$ & $\infty 2.0$ & $0 / 15$ \\
\hline & $5.0 \mathrm{e} 5$ & $6.9 \mathrm{e} 5$ & $8.9 \mathrm{e} 5$ & $1.0 \mathrm{e} 6$ & $1.1 \mathrm{e} 6$ & $1.1 \mathrm{e} 6$ & $15 / 15$ \\
\hline & $\infty$ & $\infty$ & $\infty$ & $\infty$ & $\infty$ & $\infty 2.0$ & \\
\hline )-CMA- & $\infty$ & $\infty$ & $\infty$ & $\infty$ & $\infty$ & $\infty 2.0 e 5$ & $0 / 15$ \\
\hline & $1.8 \mathrm{e} 6$ & $2.5 \mathrm{e} 6$ & $2.6 \mathrm{e} 6$ & $2.9 \mathrm{e} 6$ & $3.2 \mathrm{e} 6$ & $3.6 \mathrm{e} 6$ & $15 / 15$ \\
\hline IA-ES & $\infty$ & $\infty$ & $\infty$ & $\infty$ & $\infty$ & $\infty 2.6$ & $0 / 15$ \\
\hline )-CMA-E & $\infty$ & $\infty$ & $\infty$ & $\infty$ & $\infty$ & $\infty 2.0 e 5$ & $/ 15$ \\
\hline$f_{118}$ & 6900 & $1.2 \mathrm{e} 4$ & $1.8 \mathrm{e} 4$ & $2.6 \mathrm{e} 4$ & $3.0 \mathrm{e} 4$ & $3.3 \mathrm{e} 4$ & \\
\hline n)-CMA-ES & 1.7 & 1.7 & 1.5 & 1.5 & & 1.4 & 15 \\
\hline s)-CMA-ES & 1.2 & 1.3 & 1.1 & $1.1^{\star}$ & $1.1^{\star 3}$ & $1^{\star 3}$ & $15 / 15$ \\
\hline$f_{119}$ & 2800 & $2.9 \mathrm{e} 4$ & $3.6 \mathrm{e} 4$ & $4.1 \mathrm{e} 5$ & $1.4 \mathrm{e} 6$ & $1.9 \mathrm{e} 6$ & $\overline{15}$ \\
\hline 1)-CMA & & $\infty$ & $\infty$ & $\infty$ & $\infty$ & $\infty 2.6$ & \\
\hline )-CMA-ES & 10 & $\infty$ & $\infty$ & $\infty$ & $\infty$ & $\infty 2.0 e 5$ & $0 / 15$ \\
\hline & $3.6 \mathrm{e} 4$ & $1.8 \mathrm{e} 5$ & $2.8 \mathrm{e} 5$ & $1.6 \mathrm{e} 6$ & $6.7 \mathrm{e} 6$ & $1.4 \mathrm{e} 7$ & $13 / 15$ \\
\hline & $\infty$ & $\infty$ & $\infty$ & $\infty$ & $\infty$ & $\infty 2.0 e 5$ & \\
\hline )-CMA-I & $\infty$ & $\infty$ & $\infty$ & $\infty$ & $\infty$ & $\infty 2.0 e 5$ & $0 / 15$ \\
\hline$f_{121}$ & 250 & 770 & 1400 & 9300 & $3.4 \mathrm{e} 4$ & $5.7 \mathrm{e} 4$ & $15 / 15$ \\
\hline 1) $-\mathrm{CM}$ & 2.2 & 1.4 & 1.2 & $0.81 \downarrow 4$ & $0.8^{\downarrow} 4$ & 6.2 & $0 / 15$ \\
\hline )-CMA-ES & 1.9 & 1 & 1 & $0.58^{\star 3 \downarrow 4}$ & ${ }^{4} \mathbf{0 . 5 9} \mathbf{9}^{\star 2 \downarrow 4}$ & $4_{5.3}$ & $1 / 15$ \\
\hline$f_{122}$ & 690 & $5.2 \mathrm{e} 4$ & $1.4 \mathrm{e} 5$ & $7.9 \mathrm{e} 5$ & $2.0 \mathrm{e} 6$ & $5.8 \mathrm{e} 6$ & \\
\hline is & 45 & $\infty$ & $\infty$ & $\infty$ & $\infty$ & $\infty$ & \\
\hline )-CMA & 29 & & & $\propto$ & & $e 5$ & \\
\hline & 11 & 5.3 & $1.5 \mathrm{e} 6$ & 5.3 & 2.7 & 8 & \\
\hline 1)-CNA & & $\alpha$ & $\infty$ & $\alpha$ & $\infty$ & & \\
\hline ) $-\mathrm{CM}$ & & & $\infty$ & $\infty$ & $\infty$ & $\infty 2.0 e 5$ & \\
\hline & 190 & 2000 & $4.1 \mathrm{e} 4$ & $1.3 \mathrm{e} 5$ & $3.9 \mathrm{e} 5$ & $8.0 \mathrm{e} 5$ & $15 / 15$ \\
\hline 1)-CMA & & & $\infty$ & & $\propto$ & & \\
\hline (I) -CMA-ES & 3.5 & $1.5 \mathrm{e} 3$ & $\infty$ & $\infty$ & $\infty$ & $\infty 2.0 e 5$ & $/ 15$ \\
\hline & 1 & 1 & 1 & $2.5 \mathrm{e}^{7}$ & $8.0 \mathrm{e} 7$ & $8.1 \mathrm{e} 7$ & $1 / 15$ \\
\hline ) $-\mathrm{C}$ & 1 & & $\infty$ & $\infty$ & $\propto$ & es 5 & \\
\hline )-CMA-ES & 1 & $1.2 \mathrm{e} 5$ & $\infty$ & $\infty$ & $\infty$ & $\infty 2.0 e 5$ & $0 / 15$ \\
\hline & 1 & 1 & 1 & $\infty$ & $\infty$ & $\infty$ & \\
\hline & 1 & $\infty$ & & & $\alpha$ & $\infty$ & \\
\hline$-\mathrm{CM}$ & 1 & $\infty$ & $\infty$ & $\infty$ & $\infty$ & $\infty$ & 15 \\
\hline & 1 & 1 & 1 & $4.4 \mathrm{e} 6$ & $7.3 \mathrm{e} 6$ & $7.4 \mathrm{e} 6$ & $15 / 15$ \\
\hline n)-CMA-ES & 1 & & $\infty$ & $\infty$ & $\infty$ & $\infty 2.0 e 5$ & $0 / 15$ \\
\hline )-CMA-ES & 1 & & $\infty$ & $\infty$ & $\infty$ & 62.0e5 & \\
\hline$f_{128}$ & $1.4 \mathrm{e} 5$ & $1.3 \mathrm{e} 7$ & $1.7 \mathrm{e} 7$ & $1.7 \mathrm{e} 7$ & $1.7 \mathrm{e} 7$ & $1.7 \mathrm{e} 7$ & $9 / 15$ \\
\hline a) $-\mathrm{CMA}$ & $\infty$ & $\infty$ & $\infty$ & $\infty$ & $\infty$ & 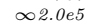 & \\
\hline )-CMA-ES & & & & $\infty$ & $\infty$ & $\infty 2.0 e 5$ & 15 \\
\hline$f_{129}$ & $7.8 \mathrm{e} 6$ & $4.1 \mathrm{e} 7$ & $4.2 \mathrm{e} 7$ & $4.2 \mathrm{e} 7$ & $4.2 \mathrm{e} 7$ & $4.2 \mathrm{e} 7$ & $\longdiv { 1 5 }$ \\
\hline 1) $-\mathrm{Cl} 1$ & $\alpha$ & & $\alpha$ & $\alpha$ & $\infty$ & & \\
\hline (s)-CMA-ES & & & $\infty$ & $\infty$ & $\infty$ & $\infty 2.0 e 5$ & $0 / 15$ \\
\hline & 4900 & & $2.5 \mathrm{e} 5$ & $2.5 \mathrm{e} 5$ & $2.6 \mathrm{e} 5$ & $2.6 \mathrm{e} 5$ & $7 / 15$ \\
\hline 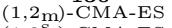 & & & 0.61 & & & 0.61 & \\
\hline & 0.95 & 0.62 & 0.34 & 0.34 & 0.34 & 0.34 & $13 / 15$ \\
\hline
\end{tabular}

Table 2: Expected running time (ERT in number of function evaluations) divided by the best ERT measured during BBOB-2009 as in Table 1 but now comparing $\left(1,2_{\mathrm{m}}\right)$-CMA-ES and $\left(1,2_{\mathrm{m}}^{\mathrm{s}}\right)$-CMA-ES. 
$5-\mathrm{D}$
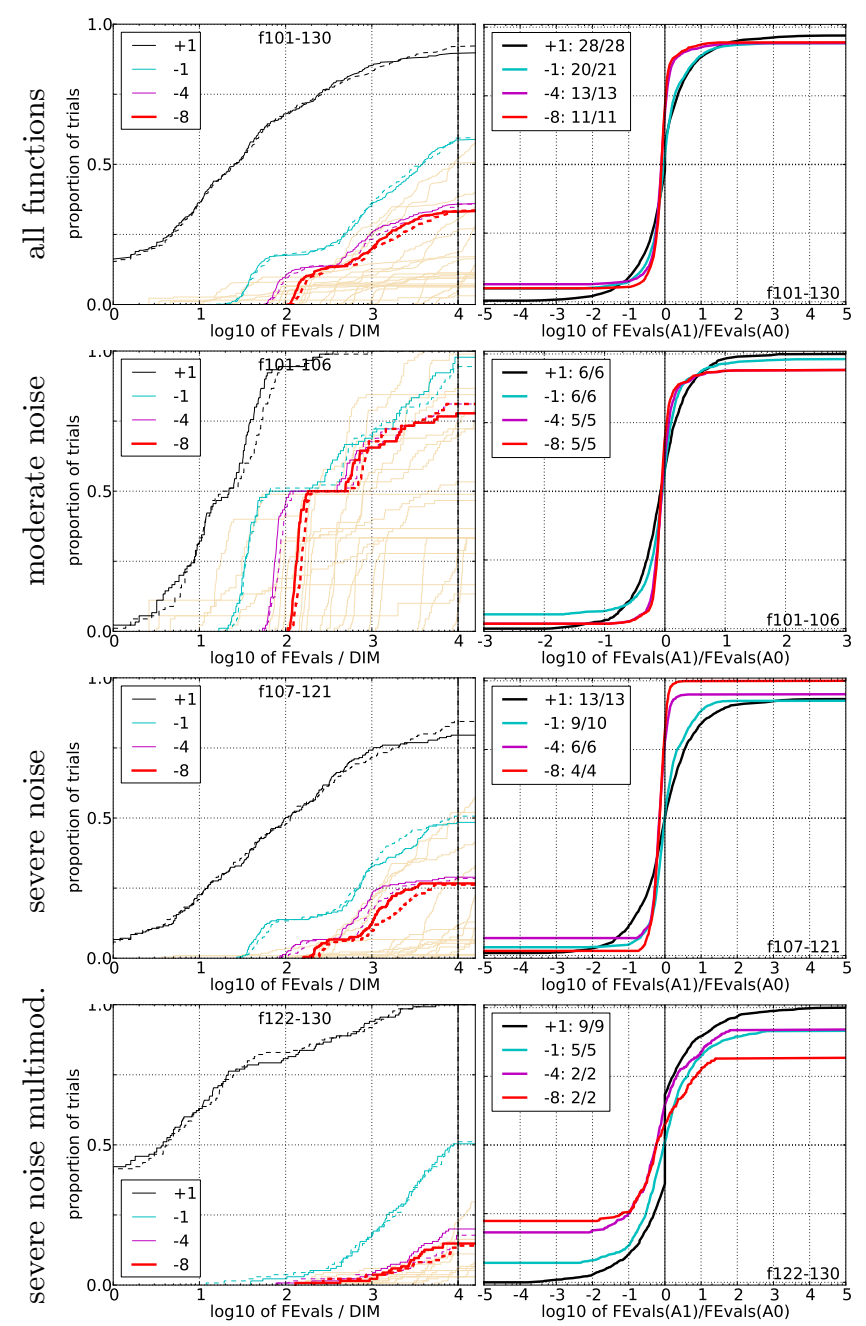

$20-\mathrm{D}$
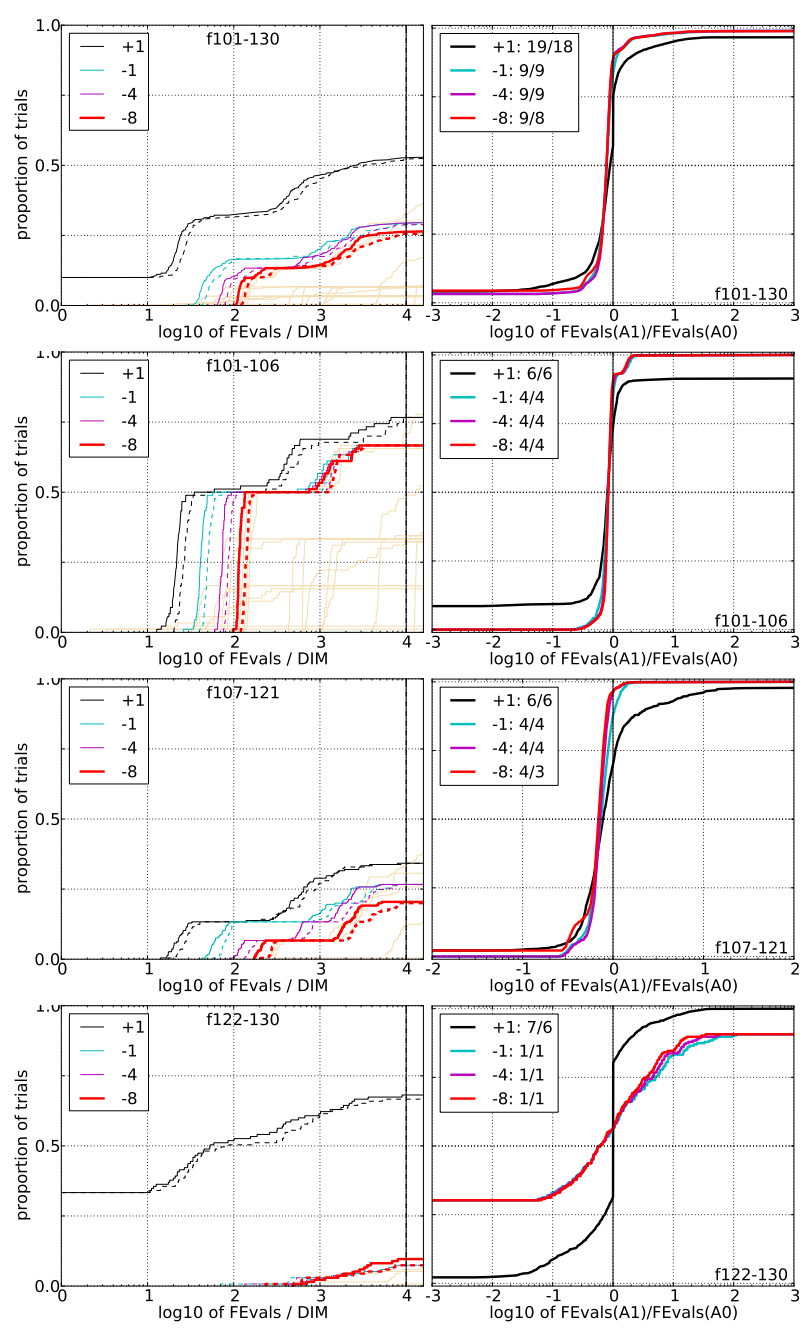

Figure 4: Empirical cumulative distributions (ECDF) of run lengths and speed-up ratios in 5-D (left) and 20-D (right) as in Fig.2 but now for $\left(1,2_{\mathrm{m}}^{\mathrm{s}}\right)$-CMA-ES (solid) and $\left(1,2_{\mathrm{m}}\right)$-CMA-ES (dashed) and ratios of $\left(1,2_{\mathrm{m}}^{\mathrm{s}}\right)$-CMA-ES divided by $\left(1,2_{\mathrm{m}}\right)$-CMA-ES respectively.

2009: Noisy functions definitions. Technical Report

RR-6869, INRIA, 2009. Updated February 2010.

[6] N. Hansen, S. D. Müller, and P. Koumoutsakos.

Reducing the time complexity of the derandomized evolution strategy with covariance matrix adaptation. Evolutionary Computation, 11(1):1-18, 2003.

[7] K. Price. Differential evolution vs. the functions of the second ICEO. In Proceedings of the IEEE International Congress on Evolutionary Computation, pages 153-157, 1997. 